\title{
On the Physical Layer Security of the Cooperative Rate-Splitting Aided Downlink in UAV Networks
}

\author{
Hamed Bastami, Mehdi Letafati, Majid Moradikia, Ahmed Abdelhadi, Senior Member, IEEE, \\ Hamid Behroozi, Member, IEEE, and Lajos Hanzo, Fellow, IEEE
}

\begin{abstract}
Unmanned Aerial Vehicles (UAVs) have found compelling applications in intelligent logistics, search and rescue as well as in air-borne Base Station (BS). However, their communications are prone to both channel errors and eavesdropping. Hence, we investigate the max-min secrecy fairness of UAVaided cellular networks, in which Cooperative Rate-Splitting (CRS) aided downlink transmissions are employed by each multiantenna UAV Base Station (UAV-BS) to safeguard the downlink of a two-user Multi-Input Single-Output (MISO) system against an external multi-antenna Eavesdropper (Eve). Realistically, only Imperfect Channel State Information (ICSI) is assumed to be available at the transmitter. Additionally, we consider a realistic total power constraint and guarantee the specific Quality of Service (QoS) requirements of the legitimate users. To handle the worst-case channel uncertainty of the legitimate users and an external Eve, we conceive a robust secure resource allocation algorithm, which maximizes the minimum worst-case secrecy rate of the legitimate users. Based on the CRS principle, the transmitter splits and encodes the messages of legitimate users into common as well as private streams and the user having stronger CSI is asked to help the cell-edge user by opportunistically forwarding its decoded common message. In contrast to the existing schemes adopted in the literature for ensuring secure transmission of the first cooperative phase only, in our proposed solution the common message has a twin-fold mission. Explicitly, apart from serving as the desired message, it also acts as Artificial Noise (AN) for drowning out Eve without consuming extra power. This is in stark contrast to the conventional AN designs. In the second phase, the pure AN is directed towards the Eve, deploying a robust Maximum Ratio Transmitter (MRT) beamformer at the UAV-BS. To solve the resultant non-convex optimization problem we resort to the Sequential Parametric Convex Approximation (SPCA) method together with a bespoke initialization algorithm to avoid any failure due to infeasibility. Our simulation results confirm that the proposed secure transmission scheme outperforms the existing cooperative benchmarkers.
\end{abstract}

Index Terms-Rate splitting, physical layer security, robust beamforming, secrecy fairness, imperfect CSIT, worst-case optimization, cellular UAV networks.

\section{INTRODUCTION}

$\mathbf{P}$ HYSICAL layer security (PLS), whose core idea is to opportunistically exploit the random characteristics of fading channels, has attracted growing attention in the recent

Hamed Bastami, Mehdi Letafati, and Hamid Behroozi are with the Department of Electrical Engineering, Sharif University of Technology, Tehran, Iran, e-mails: \{hamed.bastami@ee., mletafati@ee.,behroozi@\}sharif.edu

Majid Moradikia and Ahmed Abdelhadi are with the Engineering Technology Department at University of Houston, emails:\{mmoradikia,aabdelhadi\}@uh.edu.

Lajos Hanzo is with the University of Southampton, Southampton SO17 1BJ, U.K, e-mail: hanzo@soton.ac.uk. decade as a promising technique of enhancing the secrecy of wireless communications [1]-[10]. A pair of proposed PLS solutions, namely beamforming and jamming techniques have been particularly extensively exploited [3], [4], [6]-[9]. However, the performance of these PLS schemes critically relies on the availability of near-perfect Channel State Information at the Tx (CSIT), particularly in multi-user systems ${ }^{1}$ [10], [11].

Recently, thanks to the high mobility, on-demand coverage, and providing Line-of-Sight (LoS) link, Unmanned Aerial Vehicle (UAV) networks have attracted a great deal of interest because of their capability of supporting the Key Performance Indicators (KPI)s of next-generation networks [12], [15], [16]. In this regard, UAVs may be deployed as mobile base stations (UAV-BS) to assist the BS on the ground in its service provision. Actually, with the aim of traffic offloading [17], the burden imposed on the over-sailing macro-cell can be directed to the small UAV-cells to mitigate the burden imposed on the cellular network [12], [18]. Furthermore, UAV-BSs can assist Heterogeneous Networks (HetNet), where the problem of inter-user interference management would be problematic [12], [19]. Explicitly, to alleviate the inter-user interference within each small UAV-cell, the 3D-position of UAVs can be exploited for increasing the degrees of freedom (DoF) [20]. For achieving a high directional gain, hence minimizing the interference between the users in [21], a null-steered beamforming technique has been employed by the UAV-BSs. From a secrecy perspective, the PLS designs can also exploit the UAVs for detecting the Eves' location via the UAV-mounted camera or radar [22], as well as the new DoF provided by the trajectory design [23]-[25], and the LoS jamming link provided by the UAV-based friendly jammer [26].

Meanwhile, to satisfy the heterogeneous and massive connectivity requirements of NextG-Com, deploying Multiple Access (MA) techniques in the downlink Multi Input-Single Output Broadcast (MISO-BC) systems has been highlighted [6], [11], [12], [24], [27]-[30]. To be more specific, recently, Rate Splitting (RS) Multiple Access (RSMA), which is a generalized joint framework of Non-Orthogonal Multiple Access (NOMA) and Space Division Multiple Access (SDMA), was introduced as a promising transmission scheme, capable of outperforming both NOMA and SDMA in terms of its multiplexing gain and spectral efficiency [27]. Using RS, each transmitted message is split into a common and a private part, followed by linear transmit precoding at the multi-antenna

\footnotetext{
${ }^{1}$ There are several PLS studies that adopted their strategy based on the Eve's direction knowledge [12], [13], e.g., in airport scenarios, where the Eves might be UAVs as well [14]
} 
Tx and Successive Interference Cancellation (SIC) at each receiver [27]. As a further development, Cooperative Rate Splitting (CRS) has been studied in [28] that outperforms its Non-cooperative Rate Splitting (NRS) counterpart [29] in terms of both its reliability [27] and security [30]. To be specific, using CRS, the RS-near user having higher power with stronger CSI is asked to opportunistically forward its decoded common message to the other user. Upon considering the half-duplex constraint, data transmission takes place in two consecutive time-slots. Hence, CRS benefits from the cooperation of the legitimate users. It is worth of mentioning that as a special form of RSMA, secure NOMA systems have been proposed in [31], [32], which use AN and beamforming for secrecy purposes. However, the non-orthogonal superimposed streams, sent by the transmitter, are taken into account as "useless" in sense of secrecy, as they can be completely removed via SIC at Eve. More explicitly, the Eve is able to decode some superimposed streams and wiretap the remainder streams with less interference. In contrast, upon using RSMA approach, the common message constitutes the desired message as well as the artificial noise (AN) harnessed for confusing the potential Eve without the need for allocating extra transmit power to the AN [29], [30]. Thus, the solutions proposed by [31], [32] are not applicable to the RSMA-based counterpart owing to the different natures of the problems.

The recent CRS-enabled systems of [28], [30] have investigated the idealized simplifying assumption of having perfect CSI knowledge, but in case of UAV-communications due to instability of the platform in atmospheric turbulence, as well as its limited computation power, the CSI cannot be perfectly estimated at the UAV-BS [12], [26]. This will further exacerbate the detrimental impact of inter-user interference upon increasing the transmit power, specifically in UAV-MA networks, in which the UAV-BS is enabled by one of the MA techniques [11], [12]. Although the efficiency of RSMA has also been reported under imperfect CSIT in [11], [29], [33], little attention has been given to the integration of RSMA with UAV-BS. Explicitly, the robust and secure design of CRS-aided UAV networks, under imperfect CSIT of the whole links ${ }^{2}$, has not been studied as yet. A list of abbreviations and acronyms used throughout the paper is given in Table I. To gain more insight, the novelty of the proposed approach is boldly and explicitly contrasted to the state-of-the-art in Table II at a glance.

To elaborate our work little further, we consider a UAVaided cellular network including an over-sailing macro BS and several UAV-aided cells as well as macro-cell users. For each UAV-cell, we consider the worst-case secrecy scenario for a MISO-BC system. In particular, a multi-antenna UAV-BS aims to communicate with two legitimate users in the presence of a multi-antenna Eve, that is able to maximize its wiretapping rate by doing Maximum Ratio Combining (MRC) protocol

\footnotetext{
${ }^{2}$ Although this is more realistic to consider a completely unknown Eve, by using UAV we can detect the Eves' location and thus having a knowledge about the Eves (even imperfect E-CSIT) is reasonable. This assumption is also valid for cases in which Eves have enrolled as the network's nodes, but not authorized to receive the services, though even for a passive Eve, due to the unintentionally leakage of oscillator power, Eve's CSI can be estimated.
}

TABLE I

LIST OF ACRONYMS AND ABBREVIATIONS

\begin{tabular}{lr}
\hline PLS & Phisical Layer Security \\
FJ & Friendly Jammer \\
SIC & Successive Interference Cancellation \\
I-ESIT & Imperfect Eve's Channel State Information \\
I-ECDI & Imperfect Eve's Channel Direction Information \\
IUIM & Inter-User Interference-Management \\
RSMA & Rate-Splitting Multiple Access \\
CRS & Cooperative Rate-Splitting \\
NRS & Non-Cooperative Rate-Splitting \\
NOMA & Non-Orthogonal Multiple Access \\
SDMA & Space Division Multiple Access \\
WCSRM & Worst Case Secrecy Rate Maximization \\
SSRM & Secrecy Sum Rate Maximization \\
AN & Artificial Noise \\
ANPM & AN Power Maximization \\
SOPM & Secrecy Outage Probability Minimization \\
SRM & Secrecy Rate Maximization \\
MISO-BC & Multi-Input Single-Output Broadcast Channel \\
\hline
\end{tabular}

[7]. Against this background, the contributions of our work are summarized as follows:

- To safeguard confidentiality in the presence of multi antenna Eve, we propose a comprehensive CRS-based secrecy design, which guarantees both secrecy fairness between the two users and robustness against imperfect CSIT. To satisfy our secrecy fairness goal, given the power budget constraints imposed on our multi-carrier UAV-assisted system, we formulate the associated WorstCase Secrecy Rate (WCSR) maximization problem optimized under the practical outage constraints of both cooperative phases.

- Using the two-stage CRS strategy, we conceive a secrecy policy under which the first phase is secured by designing the RS-common-precoder, so that while Eve fails to attain a certain minimum eavesdropping rate, the common message can be decoded at legitimate users. By doing so, the common message twin-fold mission of both the information bearing intended message and the AN. Interestingly, by decoding and forwarding the common stream, two opportunities are provided to take full advantages of the common stream, hence further enhancing the secrecy. The second phase is also secured by focusing the AN towards the Eve by employing a robust Maximum Ratio Transmission (MRT) beamformer at the idle UAV-BS. Given the limited power assigned to the second phase, we also optimize the relaying and jamming power, hence further increasing the secrecy rate. To further enhance the achievable secrecy fairness, we have optimized a pair of parameters as well, i.e., weighting factor determining the specific share of each user in the secrecy enhancement as well as time-slotsharing parameter between two phases. We have also included an interference management constraint to protect the communications of over-sailing macro-cell against 
TABLE II

OVERVIEW OF EXISTING LITERATURE

\begin{tabular}{|c|c|c|c|c|c|c|c|c|c|c|c|c|c|c|c|c|c|c|c|c|}
\hline $\begin{array}{c}\text { References } \Rightarrow \\
\text { Keywords } \Downarrow \\
\end{array}$ & $\begin{array}{l}\text { Proposed } \\
\text { Approach }\end{array}$ & [3] & [4] & [7] & [8] & [9] & [12] & $\begin{array}{l}{[13]} \\
{[14]}\end{array}$ & {$[18]$} & [19] & $\begin{array}{l}{[20]} \\
{[21]}\end{array}$ & $\begin{array}{l}{[22]} \\
{[24]}\end{array}$ & [26] & [28] & [29] & [30] & [31] & [32] & [33] & [34] \\
\hline PLS & $\checkmark$ & $\checkmark$ & $\checkmark$ & $\checkmark$ & $\checkmark$ & $\checkmark$ & & $\checkmark$ & $\checkmark$ & & & $\checkmark$ & $\checkmark$ & & $\checkmark$ & $\checkmark$ & $\checkmark$ & $\checkmark$ & & $\bar{\checkmark}$ \\
\hline $\begin{array}{l}\text { Beamformer } \\
\text { (Precoder) } \\
\text { Design }\end{array}$ & $\checkmark$ & $\checkmark$ & $\checkmark$ & $\checkmark$ & $\checkmark$ & $\checkmark$ & & & & & & & & $\checkmark$ & $\checkmark$ & $\checkmark$ & $\checkmark$ & & $\checkmark$ & $\checkmark$ \\
\hline FJ Design & $\checkmark$ & & $\checkmark$ & $\checkmark$ & $\checkmark$ & $\checkmark$ & & & & & & & & & & & & & & $\checkmark$ \\
\hline Imperfect CSIT & $\checkmark$ & & & & & & $\checkmark$ & & & & & & $\checkmark$ & & & & & & $\checkmark$ & \\
\hline Unknown Eve & & & & $\checkmark$ & & & & & & & & & & & & & & & & \\
\hline I -ECSIT & $\checkmark$ & & $\checkmark$ & & & & & & & & & & $\checkmark$ & & $\checkmark$ & & $\checkmark$ & & & \\
\hline I-ECDI & & & & & $\checkmark$ & & & $\checkmark$ & & & & & & & & & & & & \\
\hline $\begin{array}{l}\text { UAV- } \\
\text { Communication }\end{array}$ & $\checkmark$ & & & & & & $\checkmark$ & & $\checkmark$ & & $\checkmark$ & $\checkmark$ & $\checkmark$ & & & & & & & \\
\hline UAV-BS & $\checkmark$ & & & & & & $\checkmark$ & & $\checkmark$ & & $\checkmark$ & & & & & & & & & \\
\hline IUIM & $\checkmark$ & & & & & & $\checkmark$ & & & $\checkmark$ & 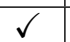 & & & & & & & & & \\
\hline UAV-HetNet & $\checkmark$ & & & & & & $\checkmark$ & & & $\checkmark$ & & & & & & & & & & \\
\hline RSMA & $\checkmark$ & & & & & & & & & & & & & $\checkmark$ & $\checkmark$ & $\checkmark$ & & & $\checkmark$ & \\
\hline CRS & $\checkmark$ & & & & & & & & & & & & & $\checkmark$ & & $\checkmark$ & & & & \\
\hline NOMA & & & & & & & $\checkmark$ & & & $\checkmark$ & & & & & & & $\checkmark$ & $\checkmark$ & & \\
\hline WCSRM & $\checkmark$ & $\checkmark$ & $\checkmark$ & & & & & & & & & & & & $\checkmark$ & & & & & \\
\hline SSRM & & & & & & & & & & & & & & & & $\checkmark$ & & & & \\
\hline ANPM & & & & $\checkmark$ & & & & & & & & & & & & & & & & \\
\hline SOPM & & & & & & 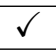 & & & & & & & & & & & & & & \\
\hline SRM & & & & & $\checkmark$ & & & & & & & & & & & & & & & $\checkmark$ \\
\hline MISO-BC & $\checkmark$ & & & & & $\checkmark$ & $\checkmark$ & & & & & & & $\checkmark$ & $\checkmark$ & $\checkmark$ & $\checkmark$ & $\checkmark$ & $\checkmark$ & \\
\hline
\end{tabular}

small UAV-cell transmissions.

- To solve the resultant non-convex optimization problem, we resort to the Sequential Parametric Convex Approximation (SPCA) methodology of [34], leading to a trade-off between approaching optimality and the computational complexity ${ }^{3}$. Moreover, to avoid any failure due to a potential infeasibiity, a beneficial initialization algorithm is proposed for finding a feasible initial point of the original problem.

- Our simulation results have shown that the proposed strategy outperforms both the Cooperative NOMA (CNOMA) and the Cooperative SDMA (C-SDMA) strategies. We will also show that although the proposed CRSbased strategy has evolved from the NRS scheme of [29] for some propagation environments, it outperforms NRS on average. More precisely, we have shown that reliance on the cooperative phase is inevitable.

The rest of this paper is organized as follows. The system model and preliminaries are provided in Section II. Section III and IV describe the signal representation and the corresponding achievable information rate, respectively. Section V formulates our robust WCSR maximization problem. The proposed SPCA-based solution converts the non-convex problem

\footnotetext{
${ }^{3}$ Notably, heuristic optimization techniques, such as GA [35], Ant Colonies [36], and Multiple-Objective GA (MOGA) [37] are capable of solving challenging rank deficient [38] and non-convex problems, even for the near-Paretooptimization of multi-component objective functions [39]. However, upon such schemes, it is often hard to provide rigorous performance guarantees.
}

into a convex problem and the associated feasible initialization procedures are provided in Section VI. In Section VII, our simulation results are presented and the paper is concluded in Section VIII. Finally, the Appendices and Proofs of the lemmas are provided in Section IX.

Notation: Vectors and matrices are denoted by lower-case and upper-case boldface symbols, respectively; $(.)^{\mathrm{T}},(.)^{*},(.)^{\mathrm{H}}$, and $(.)^{-1}$ denote the transpose, conjugate, conjugate transpose, and inverse of a matrix respectively; $\mathfrak{R} e($.$) denote the real$ part of a complex variable, and $\Im m($.$) denote the imaginary$ part of a complex variable; We use $\mathbb{E}\{\cdot\}$ and $\triangleq$ to denote the expectation and definition operations, respectively; A complex Gaussian random variable with mean $\mu$ and variance $\sigma^{2}$ reads as $\mathcal{C N}\left(\mu, \sigma^{2}\right)$. The notation $\operatorname{Vec}(\mathbf{H})$ converts the matrix $\mathbf{H}$ in single column vector, and $\mathbf{I}_{N}$ denotes the $N \times N$ identity matrix; $\mathbb{R}^{N \times 1}$ and $\mathbb{C}^{N \times 1}$ denote the set of $N$-dimensional standard real and complex Gaussian random variable, respectively. $\mathbb{C}^{N \times N}$ stands for an $N \times N$ element standard complex Gaussian random matrix whose real and imaginary parts are independent normally distributed random variables with mean zero and variance $\frac{1}{2}$. The entry in the $i$-th row and $j$-th column of a matrix $\mathbf{H}$ is represented by $\mathbf{H}[i, j]$. Finally, $\mathcal{D}(\mathbf{H})$ denotes the dimensions of the matrix $\mathbf{H}$ given by the number of rows and by the number of columns. Furthermore, $\mathbf{u}_{\max }\{\mathbf{A}\}$ and $\mathbf{v}_{\max }\{\mathbf{A}\}$ denote the columns of $\mathbf{U}_{\mathbf{A}}$ and $\mathbf{V}_{\mathbf{A}}$ corresponding to the dominant singular value $\sigma_{\max }$ of matrix A, respectively, i.e., the matrix $\mathbf{A}$ has a Singular Value 


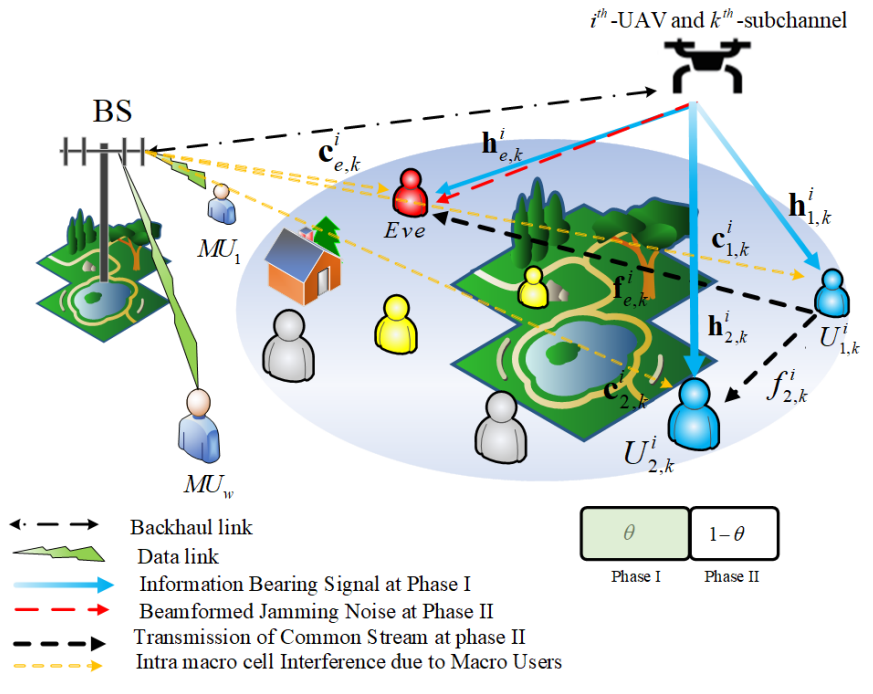

Fig. 1. Proposed system model

Decomposition (SVD) given by $\mathbf{A} \triangleq \mathbf{U}_{\mathbf{A}} \boldsymbol{\Lambda}_{\mathbf{A}} \mathbf{V}_{\mathbf{A}}$.

\section{System Model AND PRELIMINARIES}

In this section, the RS signal model, the channel models and their corresponding realistic impaired counterparts are firstly reviewed, which then serve as the basis of developing a robust and secure approach in an RS-based UAV-assisted cellular network. In this regard, a network including a over-sailing macro BS and $I$ UAV cells as well as $N_{M}$ macro users are considered, as shown in Fig. 1. Although the macro users have higher priority in using the spectrum, the UAVs have been allowed by the BS to operate within the service range of the macro users. For the $i$-th UAV cell, a UAV hovering at a fixed altitude $H_{i}$ provides communication services for $N^{i}$ number of UAV users. The total bandwidth of $B W$ is shared among all UAV users. The same spectrum is exploited by the macrocell users and all the UAV cells, so the nodes outside the $i$-th UAV cell may interfere with the UAV nodes therein, inflicting Intra Macro-Cell Interference (IMCI). For each UAV cell, $B W$ is partitioned into $K$ sub-channels $\left.S C_{k}\right|_{k=1} ^{K}$, each of which has a bandwidth of $B_{s c}=\frac{B W}{K}$. If we let $\left.N_{k}\right|_{k=1} ^{K}$ stand for the number of users assigned to $S C_{k}$, a maximum number of $N^{i}=\sum_{k=1}^{K} N_{k}$ users can be served by $U A V_{i}$. However, without loss of generality we assume $N_{k}=2$ in the rest of this paper.

\section{A. Transmitted RS Signal Model}

We consider the $i$-th UAV cell ${ }^{4}$ of Fig. 1 , where $U A V_{i}$ aims to communicate with two legitimate users, namely $U_{1, k}^{i}$ and $U_{2, k}^{i}$ through $S C_{k}$, while Eve keeps on wiretapping silently. $U A V_{i}$ and Eve are multi-antenna devices respectively have $N_{t}$ Transmit Antennas (TAs) and $N_{e}$ Receive Antenna (RAs), while $U_{1, k}^{i}$ and $U_{2, k}^{i}$ are equipped with a single RA.

To concurrently serve two users through $S C_{k}, U A V_{i}$ relies on the RS technique as shown if Fig. 2, which partitions

\footnotetext{
${ }^{4}$ Although the analysis is provided for a single UAV cell, the results can be generalized for the whole network.
}

the confidential messages destined for each legitimate user $\left.\mathcal{W}_{n, k}^{i}\right|_{n=1} ^{2}$ into the common part $\mathcal{W}_{n, k}^{i, c}$, and private part $\mathcal{W}_{n, k}^{i, p}$. The resultant common parts $\left.\mathcal{W}_{n, k}^{i, c}\right|_{n=1} ^{2}$ are incorporated into the $\mathcal{W}_{k}^{i, c}$, and encode to the common stream $s_{k}^{i, c}$. By contrast, $\mathcal{W}_{1, k}^{i, p}$ and $\mathcal{W}_{2, k}^{i, p}$ are independently encoded into two different private streams $s_{1, k}^{i, p}, s_{2, k}^{i, p}$, respectively. Upon grouping the three streams into the vector $\mathbf{s}_{k}^{i} \triangleq\left[s_{k}^{i, c}, s_{1, k}^{i, p}, s_{2, k}^{i, p}\right]^{\mathrm{T}}$, the transmitted RS signal is given by:

$$
\mathbf{x}_{k}^{i}=\mathbf{P}_{k}^{i} \mathbf{s}_{k}^{i}=\mathbf{p}_{k}^{i, c} s_{k}^{i, c}+\sum_{n=1}^{N_{k}} \mathbf{p}_{n, k}^{i, p} s_{n, k}^{i, p},
$$

where $\mathbf{P}_{k}^{i}=\left[\mathbf{p}_{k}^{i, c}, \mathbf{p}_{1, k}^{i, p}, \mathbf{p}_{2, k}^{i, p}\right] \in \mathbb{C}^{N_{t} \times 3}$ is the beamforming matrix adopted by $U A V_{i}$, which includes the linear precoding vector $\mathbf{p}_{k}^{i, c}$, corresponding to the common stream as well as the precoding vectors $\mathbf{p}_{1, k}^{i, p}, \mathbf{p}_{2, k}^{i, p}$ of the private streams. As for the power constraints, on one hand the RS signal's transmit power $p_{k}^{i} \triangleq \operatorname{Tr}\left(\mathbf{x}_{k}^{i} \mathbf{x}_{k}^{i}{ }^{H}\right)$, has to satisfy the power constraint budget of $S C_{k}$, i.e. $\bar{P}_{k}^{i}$. On the other hand, the power budget assigned to different $S C_{k}$ has to meet the power limit $\bar{P}_{U A V}^{i}$ at $U A V_{i}$. Assuming that $\mathbb{E}\left\{\mathbf{s}_{k}^{i} \mathbf{s}_{k}^{i^{H}}\right\}=\mathbf{I}_{3}$, these constraints can be expressed as:

$$
\begin{gathered}
0 \leq \operatorname{Tr}\left(\mathbf{P}_{k}^{i} \mathbf{P}_{k}^{i H}\right) \leq \bar{P}_{k}^{i}, \forall k=1, . ., K \\
\sum_{k=1}^{K} \bar{P}_{k}^{i} \leq \bar{P}_{U A V}^{i} .
\end{gathered}
$$

At the receiver side, each user first reconstructs its corresponding message by decoding $s_{k}^{i, c}$ to retrieve the associated common message $\mathcal{W}_{n, k}^{i, c}$, followed by decoding the associated private streams by employing a different codebook from the one used at the transmitter [11].

We assume furthermore that, the received power of the cellcenter user $U_{1, k}^{i}$ is higher than that of the cell-edge user $U_{2, k}^{i}$. So, to further assist the transmission of $U_{2, k}^{i}$, the cell-center user $U_{1, k}^{i}$ acts as a Decode-and-Forward (DF) relaying user who re-encodes $s_{k}^{i, c}$ and forwards it to $U_{2, k}^{i}$. Given that all nodes obey the half-duplex constraint, data transmission takes place in two consecutive time-slots. Consequently, a dynamic time sharing parameter $0 \leq \theta \leq 1$ is introduced, where the portion $\theta$ of each time slot is allocated for the first phase of transmissions, while the relaying phase is allocated to the remaining $(1-\theta)$ portion. Thus, two opportunities are provided for Eve to wiretap the information during these two phases of transmissions. To safeguard the first phase, the corresponding beamformer $\mathbf{p}_{k}^{i, c}$ of the common stream is designed so that $s_{k}^{i, c}$ is simultaneously exploited as jamming noise at Eve, as detailed later in the paper. By contrast, in order to secure the transmission against Eve during the relaying phase, $U A V_{i}$ serves as a jammer. It should be emphasized that, we here assume the worst-case scenario in the sense of security, where the powerful, and high-complexity receiver of Eve knows its exact CSI and can use Maximum Ratio Combining (MRC) and wiretap for gleaning confidential information at its full capacity. 


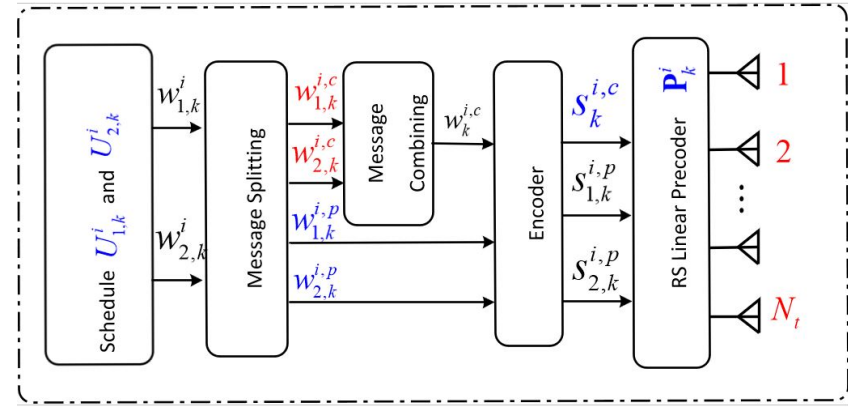

Fig. 2. Rate splitting transmission Structure

\section{B. Channel Definitions}

The complex-valued channel coefficients between the $\mathrm{UAV}$ and the terrestrial nodes $\mathrm{UAV}_{i} \rightarrow U_{1, k}^{i}, \mathrm{UAV}_{i} \rightarrow$ $U_{2, k}^{i}, \mathrm{UAV}_{i} \rightarrow E v e$ at the first phase are denoted by $\mathbf{h}_{n, k}^{i} \in$ $\mathbb{C}^{N_{t} \times 1}, n \in\{1,2\}$ and $\mathbf{H}_{e, k}^{i} \in \mathbb{C}^{N_{e} \times N_{t}}$, respectively. These channels are modeled as $\mathbf{h}_{n, k}^{i}=\mathrm{PL}_{n . k}^{i} \mathbf{n}_{n, k}^{i}, \forall n \in\{1,2, e\}$, with $\mathbf{h}_{e, k}^{i} \triangleq \operatorname{Vec}\left(\mathbf{H}_{e, k}^{i}\right) \in \mathbb{C}^{N_{t} N_{e} \times 1}$, and $\mathrm{PL}_{n . k}^{i} \triangleq d_{n, i}^{-\alpha_{n, k}^{i}}$ represents the path loss between $U A V_{i}$ and node $n \in\{1,2, e\}$, which is characterized by the distance between $U A V_{i}$ and a ground user. Furthermore, $\mathbf{n}_{n, k}^{i}$ is i.i.d. Gaussian random variable; namely, $\mathbf{n}_{n, k}^{i}[i] \sim \mathcal{C N}(0,1)$ represents the corresponding small scale fading. The path loss exponent $\alpha_{n, k}^{i}$ obeys the probabilistic model of [40], which is appropriate for low-altitude UAVs including the LOS link that may be blocked by various environmental obstacles, given by:

$$
\alpha_{n, k}^{i} \triangleq \frac{\mathcal{L}_{n, k}^{i}-\mathcal{N}_{n, k}^{i}}{1+\lambda_{1} e^{\lambda_{2}\left(\phi_{n}^{i}-\lambda_{1}\right)}}+\mathcal{N}_{n, k}^{i} .
$$

In fact, $\alpha_{n, k}^{i}$ is composed of the LoS and non-LoS components $\mathcal{L}_{n, k}^{i}$ and $\mathcal{N}_{n, k}^{i}$, respectively. Furthermore, $\phi_{n}^{i} \triangleq$ $\frac{180}{\pi} \sin ^{-1}\left(\frac{H_{i}}{d_{n}^{i}}\right)$, denotes the elevation angle between $U A V_{i}$ and the $n$-th user at a UAV to ground distance $d$, while $\lambda_{1}$ and $\lambda_{2}$ are determined by the wireless environment, for example urban, dense urban, etc. [40].

Additionally, $\mathbf{f}_{e, k}^{i} \in \mathbb{C}^{N_{e} \times 1}$ and $f_{2, k}^{i} \sim \in \mathbb{C}^{1 \times 1}$ respectively denote the corresponding channel coefficients of $U_{1, k}^{i} \rightarrow E v e$ and $U_{1, k}^{i} \rightarrow U_{2, k}^{i}$ links during the second phase. Furthermore, the transmissions of all nodes, relying on $S C_{k}$ and within the $i$-th UAV cell, are contaminated during both phases by the IMCI signal $\boldsymbol{v}_{k}^{i} \in \mathbb{C}^{N_{M} \times 1}$ (with $N_{M}$ representing the number of macro users, occupying the same $S C_{k}$ ) and the corresponding channels are denoted by $\mathbf{c}_{n, k}^{i} \in \mathbb{C}^{N_{M} \times 1}$ for $n \in\{1,2\}$ and $\mathbf{C}_{e, k}^{i} \in \mathbb{C}^{N_{M} \times N_{e}}$.

Remark 1. (Channel Imperfection): In a realistic system, due to the transmission and processing delays, as well as the mobility of the users, the CSI may become outdated, hence resulting in inaccurate CSI that must be taken into account in a practical design. However, the path loss changes slowly, hence we assume that it can be perfectly compensated by power control. Thus, in this paper it is assumed that the CSI uncertainty is only affected by the small scale fading component $\mathbf{n}_{n, k}^{i}$. To be specific, because of the UAV's platform fluctuations due to atmospheric turbulence, as well as owing to the limited computational power of the UAVs as compared to the BSs on the ground, the CSI error is assumed to contaminate the UAV cell's links.

Based on Remark 1 together with the worst-case CSI error model of [29], [41], the relationship between the estimated small scale fading coefficients, i.e. $\hat{\mathbf{n}}_{n, k}^{i}, \hat{\mathbf{f}}_{e, k}^{i}, \hat{f}_{2, k}^{i}$ and their corresponding exact counterparts, i.e. $\mathbf{n}_{n, k}^{i}, \mathbf{f}_{e, k}^{i}, f_{2, k}^{i}$, $\forall n \in\{1,2, e\}$ becomes:

$$
\begin{gathered}
\mathbf{n}_{n, k}^{i} \triangleq \hat{\mathbf{n}}_{n, k}^{i}+\triangle \mathbf{n}_{n, k}^{i}, \\
f_{2, k}^{i} \triangleq \hat{f}_{2, k}^{i}+\triangle f_{2, k}^{i}, \mathbf{f}_{e, k}^{i} \triangleq \hat{\mathbf{f}}_{e, k}^{i}+\triangle \mathbf{f}_{e, k}^{i}, \\
\Theta_{\mathbf{h}_{n}}=\left\{\triangle \mathbf{n}_{n, k}^{i} \in \mathbb{C}^{N_{t} \times 1}:\left\|\triangle \mathbf{n}_{n, k}^{i}\right\|^{2} \leq \mathcal{D}\left(\mathbf{n}_{n, k}^{i}\right) \zeta^{2}\right\} \\
\Theta_{f_{2}}=\left\{\triangle f_{2, k}^{i}:\left|\triangle f_{2, k}^{i}\right|^{2} \leq \zeta^{2}\right\}, \\
\Theta_{\mathbf{f}_{e}}=\left\{\triangle \mathbf{f}_{e, k}^{i}:\left|\triangle \mathbf{f}_{e, k}^{i}\right|^{2} \leq \mathcal{D}\left(\mathbf{f}_{e, k}^{i}\right) \zeta^{2}\right\},
\end{gathered}
$$

where $\triangle \mathbf{n}_{n, k}^{i}, \triangle \mathbf{f}_{e, k}^{i}$, and $\triangle f_{2, k}^{i}$ respectively stand for the CSI error corresponding to $\mathbf{n}_{n, k}^{i}, \mathbf{f}_{e, k}^{i}$, and $f_{2, k}^{i}$. As seen in (6), $\zeta$ specifies the radius of the uncertainty regions of $\triangle f_{2, k}^{i}$, whilst for the vector of $\triangle \mathbf{n}_{n, k}^{i}$ and $\triangle \mathbf{f}_{e, k}^{i}$, the error are region bounded by the radius of $\sqrt{\mathcal{D}\left(\mathbf{n}_{n, k}^{i}\right)} \zeta$ and $\sqrt{\mathcal{D}\left(\mathbf{f}_{e, k}^{i}\right)} \zeta$, respectively.

\section{Signal Representation}

In this section, we firstly drive the channel capacities, which are then used in the next sub-section to calculate the Effective Secrecy Throughput (EST), which is a well-suited performance metric in the face of channel uncertainties. Before proceeding, Fig. 3 shows the flow of the analysis described in the sequel. In terms of the worst-case secrecy scenario, Eve is assumed to be able to perfectly estimate its corresponding CSI. As for the legitimate UAV-Tx, only imperfect CSIT of the links is available at the Tx. Given this perspective, while Eve can achieve the actual channel capacity, Tx is only capable of achieving the estimated information rate. Thus we first derive the actual channel capacities and then obtain the estimated information rates, as shown in Fig. 3, as well.

\section{A. Actual Channel Capacity}

As discussed earlier, during the first time slot, $U A V_{i}$ transmits the RS signal $\mathbf{x}_{k}^{i}$. Accordingly, using the actual channel parameters, the signal received at $U_{1, k}^{i}, U_{2, k}^{i}$, and Eve in the first phase, respectively, which are denoted by $y_{1, k}^{i}{ }^{(1)}, y_{2, k}^{i}{ }^{(1)}$, and $y_{e, k}^{i}{ }^{(1)}$ are obtained as follows:

$$
\begin{gathered}
y_{n, k}^{i}{ }^{(1)}=\mathbf{h}_{n, k}^{i}{ }^{H} \mathbf{x}_{k}^{i}+\sqrt{p_{M, k}} \mathbf{c}_{n, k}^{i}{ }^{H} \mathbf{v}_{k}^{i}+z_{n, k}^{i}{ }^{(1)}, \forall n \in\{1,2\} \\
y_{e, k}^{i{ }^{(1)}}=\mathbf{w}_{e, k}^{(1)^{H}}\left(\mathbf{H}_{e, k}^{i} \mathbf{x}_{k}^{i}+\sqrt{p_{M, k}} \mathbf{C}_{e, k}^{i}{ }^{H} \mathbf{v}_{k}^{i}+\mathbf{z}_{e, k}^{i}{ }^{(1)}\right),
\end{gathered}
$$

where $\mathbf{w}_{e, k}^{(1)} \triangleq \mathbf{u}_{\max }\left\{\mathbf{G}_{e, k}\right\}$ represents the MRC beamformer employed by Eve during the first phase, $\mathbf{G}_{e, k} \triangleq$ $\mathbf{H}_{e, k}^{i}\left(\mathbf{H}_{e, k}^{i}\right)^{H}$, and the term $\mathbf{c}_{n, k}^{i}{ }^{H} \mathbf{v}_{k}^{i}$ appears as a result of 


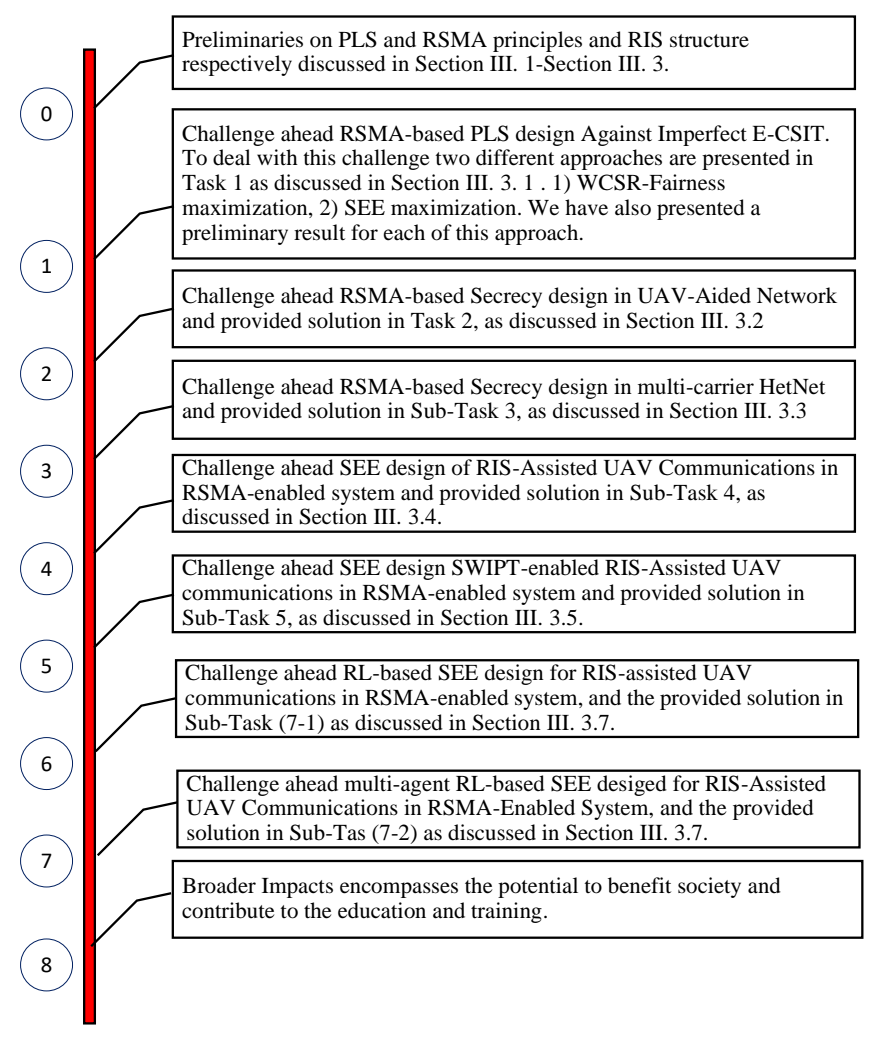

Fig. 3. Flow of the mathematical analysis

IMCI and we also readily assume that all the $N_{M}$ macro users, within on $S C_{k}$, are allocated the same power $p_{M, k}$.

As mentioned above, in Phase II, while the relaying user $U_{1, k}^{i}$ forwards the re-encoded common stream $s_{k}^{i, c}$ by employing a codebook different from that of $U A V_{i}$ to $U_{2, k}^{i}$ at a power level $0 \leq p_{R} \leq \bar{P}_{R}, U A V_{i}$ transmits the AN at a power of $0 \leq p_{J} \leq \bar{P}_{J}$. Given the limited power budget $\bar{P}^{(2)}$ in Phase II, i.e. $0 \leq p_{R}+p_{J} \leq \bar{P}^{(2)}$, it should be optimally shared between the Tx $U_{1, k}^{i}$ and the jammer $U A V_{i}$ for further enhancing the secrecy performance. For AN transmission, we use the estimated channel coefficients and then the MRT-based beamformer $\hat{\mathbf{p}}_{z} \triangleq \mathbf{v}_{\max }\left\{\hat{\mathbf{G}}_{e, k}^{H}\right\}$, where $\hat{\mathbf{G}}_{e, k}^{H} \triangleq\left(\hat{\mathbf{H}}_{e, k}^{i}\right)^{H} \hat{\mathbf{H}}_{e, k}^{i}$, is adopted by $U A V_{i}$ to focus the AN $z$ on the $E v e^{5}$. Accordingly, the signals received by $U_{1, k}^{i}$ and Eve in Phase II, are respectively written as follows:

$$
\begin{aligned}
y_{2, k}^{i}{ }^{(2)}= & \sqrt{p_{R}} f_{2, k}^{i}{ }^{H} s_{k}^{i, c}+\sqrt{p_{j}} \mathbf{h}_{2, k}^{i}{ }^{H} \hat{\mathbf{p}}_{z} z+ \\
& \sqrt{p_{M, k}} \mathbf{c}_{2, k}^{M{ }^{M} H} \mathbf{v}_{k}^{i}+z_{2, k}^{i}{ }^{(2)},
\end{aligned}
$$

\footnotetext{
${ }^{5}$ Note that, in addition to optimal power allocation (OPA) between jamming and data signals, an appropriate selection of the beamforming vector affects the secrecy performance and thus it would be nice to look for an optimal beamformer. However, this will lead to an intractable PLS design and we left it for future work.
}

$$
\begin{aligned}
y_{e, k}^{i(2)}= & \mathbf{w}_{e, k}^{(2)}{ }^{H}\left(\sqrt{p_{R}} \mathbf{f}_{e, k}^{i}{ }^{H} s_{k}^{i, c}+\sqrt{p_{j}} \mathbf{H}_{e, k}^{i} \hat{\mathbf{p}}_{z} z\right. \\
& \left.+\sqrt{p_{M, k}} \mathbf{C}_{2, k}^{M, i}{ }^{H} \mathbf{v}_{k}^{i}+\mathbf{z}_{e, k}^{i}{ }^{(2)}\right)
\end{aligned}
$$

where $\mathbf{w}_{e, k}^{(2)} \triangleq \frac{\mathbf{f}_{e, k}^{i}{ }^{H}}{\left\|\mathbf{f}_{e, k}^{i}\right\|}$ is the MRC beamformer employed by Eve during the second phase, $\mathbf{z}_{e, k}^{i}{ }^{(2)} \sim \mathcal{C N}\left(0, \delta^{2} \mathbf{I}_{N_{e}}\right)$, and $\left.z_{2, k}^{i}{ }^{(m)}\right|_{m=1} ^{2} \sim \mathcal{C N}\left(0, \delta^{2}\right)$ represent the Additive White Gaussian Noise (AWGN).

The corresponding capacity achieved by each of $U_{1, k}^{i}, U_{2, k}^{i}$, and Eve is determined by their SINR values in both phases. To do so, we should point out that in Phase I, both $U_{1, k}^{i}$ and $U_{2, k}^{i}$ first detects $s_{k}^{i, c}$, while treating the other streams as interference. Then, $s_{k}^{i, c}$ is eliminated via the SIC and each of $U_{1, k}^{i}, U_{2, k}^{i}$ can detect the corresponding private streams, while treating the irrelevant private streams as noise. As for Eve, we should note that a persistent Eve may succeed in stealing the codebooks and extract the private messages. So, an interesting PLS approach is to degrade the ability of Eve to detect the common stream at an earlier stage so that $s_{k}^{i, c}$ behaves as AN at Eve.

Given the above model, for each $n \in\{1,2, e\}$ we first let $\gamma_{n, k}^{i, c^{(1)}}$ and $\gamma_{n, k}^{i, c^{(2)}}$ respectively represent the received SINR values of users during detecting the common stream in both phases and $\gamma_{n, k}^{i, p}$ represents the SINR values at the legitimate users, when detecting the private streams. We also assume $\gamma_{e, n, k}^{i, p}$ to be the SINR at Eve when eavesdropping on the associated private stream of $U_{n, k}^{i}$, while treating the private steam of other user $\left.U_{j, k}^{i}\right|_{j \neq n}$ as interference. The corresponding capacity parameters are defined by simply replacing $\gamma$ with $C$, leading to the achievable capacity corresponding to the SINRs, respectively, which are denoted by $C_{n, k}^{i, c}(1), C_{n, k}^{i, c}{ }^{(2)}, C_{n, k}^{i, p}$, $C_{e, n, k}^{i, p}$. Subsequently, the corresponding capacity achieved by the legitimate users and Eve are respectively formulated as follows:

$$
\begin{aligned}
& C_{n, k}^{i, c}(1) \triangleq \theta \log _{2}\left(1+\gamma_{n, k}^{i, c(1)}\right), \forall n \in\{1,2, e\} \\
& C_{n, k}^{i, c(2)} \triangleq(1-\theta) \log _{2}\left(1+\gamma_{n, k}^{i, c(2)}\right), \forall n \in\{2, e\} \\
& C_{u, k}^{i, c} \triangleq \min \{\underbrace{C_{1, k}^{i, c}(1)}_{C_{1, k}^{i, c}}, \underbrace{C_{2, k}^{i, c}(1)+C_{2, k}^{i, c}(2)}_{C_{2, k}^{i, c}}\}, \\
& C_{e, k}^{i, c} \triangleq C_{e, k}^{i, c}(1)+C_{e, k}^{i, c}(2), \\
& C_{n, k}^{i, p} \triangleq \theta \log _{2}\left(1+\gamma_{n, k}^{i, p}\right), C_{e, n, k}^{i, p} \triangleq \theta \log _{2}\left(1+\gamma_{e, n, k}^{i, p}\right) \text {, }
\end{aligned}
$$

$\forall n \in\{1,2\}$ where $C_{u, k}^{i, c}$ and $C_{e, k}^{i, c}$, respectively, stand for the information capacity achieved by the legitimate users and Eve in detecting $s_{k}^{i, c}$ during the two phases. Note that, $C_{u, k}^{i, c}$ is limited by the achievable capacity of the worst-case user, i.e. the one having the lowest capacity during the two phases and Eve tries to improve the decodability of $s_{k}^{c}$ by exploiting the achievable capacities of both phases, as shown in (15). Ad- 
ditionally, $C_{n, k}^{i, c}$ represents the information capacity achieved by each of $U_{1, k}^{i}, U_{2, k}^{i}$ in detecting their corresponding private streams. Furthermore, $C_{e, n, k}^{i, p}$ denotes the capacity achieved by Eve in her attempt to detect the private stream, belonging to each legitimate user. In this case, it should be observed that, in contrast to the detection process of the private streams at $U_{1, k}^{i}$ and $U_{2, k}^{i}$, when Eve attempts to extract the associated private message of each user, the common stream $s_{k}^{i, c}$ is taken into account as interference. Similarly, the other user's private stream also acts as interference.

In accordance with the received signal models (9) and (10), the SINR values used in (12)-(16) are calculated as follows $\forall n \in\{1,2\}$ :

$$
\begin{aligned}
& \gamma_{n, k}^{i, \mathrm{c}(1)}=\frac{\left|\mathbf{h}_{n, k}^{i}{ }^{H} \mathbf{p}_{k}^{i, c}\right|^{2}}{\sum_{l=1}^{2}\left|\mathbf{h}_{n, k}^{i}{ }^{H} \mathbf{p}_{l, k}^{i, p}\right|^{2}+p_{M, k}\left\|\mathbf{c}_{n, k}^{M, i}\right\|^{2}+\delta^{2}}, \\
& \gamma_{\mathrm{e}, k}^{i, \mathrm{c}(1)}=\frac{\left|\mathbf{h}_{e, k}^{(1)}{ }^{H} \mathbf{p}_{k}^{i, c}\right|^{2}}{\sum_{i=1}^{I}\left|\mathbf{h}_{e, k}^{(1)}{ }^{H} \mathbf{p}_{l, k}^{i, p}\right|^{2}+p_{M, k}\left\|\mathbf{w}_{e, k}^{(1)}{ }^{H} \mathbf{C}_{e, k}^{M, i}\right\|^{2}+\delta^{2}}, \\
& \gamma_{2, k}^{i, c}{ }^{(2)}=\frac{p_{R}\left|f_{2, k}^{i}\right|^{2}}{p_{j}\left|\mathbf{h}_{2, k}^{i}{ }^{H} \hat{\mathbf{p}}_{z}\right|^{2}+p_{M, k}\left\|\mathbf{c}_{2, k}^{M, i}\right\|^{2}+\delta^{2}}, \\
& \gamma_{e, k}^{i, c}(2)=\frac{p_{R}\left|\mathbf{w}_{e, k}^{(2)}{ }^{H} \mathbf{f}_{e, k}^{i}\right|^{2}}{p_{j}\left|\mathbf{w}_{e, k}^{(2)}{ }^{H} \hat{\mathbf{p}}_{z}\right|^{2}+p_{M, k}\left\|\mathbf{w}_{e, k}^{(2)^{H}} \mathbf{C}_{e, k}^{M, i}\right\|^{2}+\delta^{2}}, \\
& \gamma_{n, k}^{i, \mathrm{p}}=\frac{\left|\mathbf{h}_{n, k}^{i}{ }^{H} \mathbf{p}_{n, k}^{i, p}\right|^{2}}{\left|\mathbf{h}_{n, k}^{i}{ }^{H} \mathbf{p}_{j, k}^{i, p}\right|^{2}+p_{M, k}\left\|\mathbf{c}_{n, k}^{M, i}\right\|^{2}+\delta^{2}}, n \neq j, \\
& \forall n, j \in\{1,2\}
\end{aligned}
$$

$$
\gamma_{e, n, k}^{i, \mathrm{p}}=
$$$$
\frac{\left|\mathbf{h}_{e, k}^{(1)} \mathbf{p}_{n, k}^{i, p}\right|^{2}}{\left|\mathbf{h}_{e, k}^{(1)} \mathbf{p}_{k}^{i, c}\right|+\left|\mathbf{h}_{e, k}^{(1)} \mathbf{p}_{j, k}^{i, p}\right|^{2}+p_{M, k}\left\|\mathbf{w}_{e, k}^{(1)}{ }^{H} \mathbf{C}_{e, k}^{M, i}\right\|^{2}+\delta^{2}}
$$

where $n \neq j, \forall n, j \in\{1,2\}$ and $\mathbf{h}_{e, k}^{(1)} \triangleq \mathbf{H}_{e, k}^{i}{ }^{H} \mathbf{w}_{e, k}^{(1)}$.

\section{B. Achievable Information Rate}

In order to determine the estimated SINR values, the actual channel parameters $\mathbf{h}_{n, k}^{i}, \mathbf{f}_{e, k}^{i}$, and $f_{2, k}^{i}$ in (17)- (22) can be readily substituted by their estimated counterparts of $\hat{\mathbf{h}}_{n, k}^{i}=$
$\mathrm{PL}_{n . k}^{i} \hat{\mathbf{n}}_{n, k}^{i}, \hat{\mathbf{f}}_{e, k}^{i}, \hat{f}_{2, k}^{i}$, resulting in the estimated SINR values $\hat{\gamma}_{n, k}^{i, \mathrm{c}(1)}, \hat{\gamma}_{n, k}^{i, \mathrm{c}(2)}, \hat{\gamma}_{n, k}^{i, \mathrm{p}}, \hat{\gamma}_{e, n, k}^{i, \mathrm{p}}$.

Remark 2. Given the CSI uncertainty, the link's Outage Probability (OP) might be increased. As a result the EST criterion, which is a function of both the Achievable Information Rate (AIR) as well as of the Non-zero OP (NOP), is considered.

Then, the Estimated Achievable Information Rate (EAIR ${ }^{6}$ ) corresponding to each legitimate user as well as Eve, (the achievable capacities from the Tx viewpoint), can be written as:

$$
\begin{aligned}
& \hat{R}_{n, k}^{i, c(1)}=\theta \log _{2}\left(1+\hat{\gamma}_{n, k}^{i, \mathrm{c}(1)}\right), \forall n \in\{1,2, e\} \\
& \hat{R}_{n, k}^{i, c}{ }^{(2)}=(1-\theta) \log _{2}\left(1+\hat{\gamma}_{n, k}^{i, \mathrm{c}(2)}\right), \forall n \in\{2, e\} \\
& \hat{R}_{u, k}^{i, c}=\left(1-\epsilon_{\text {out }}^{\max }\right) \min \{\underbrace{\hat{R}_{1, k}^{i, c}(1)}_{\hat{R}_{1, k}^{i, c}}, \underbrace{\hat{R}_{2, k}^{i, c(1)}+\hat{R}_{2, k}^{i, c}(2)}_{\hat{R}_{2, k}^{i, c}}\} \text {, } \\
& \hat{R}_{e, k}^{i, c} \triangleq \hat{R}_{e, k}^{i, c(1)}+\hat{R}_{e, k}^{i, c(2)}, \\
& \hat{R}_{n, k}^{i, p}=\left(1-\epsilon_{\text {out }}^{\max }\right) \theta \log _{2}\left(1+\hat{\gamma}_{n, k}^{i, p}\right), \\
& \forall n \in\{1,2\} \\
& \hat{R}_{e, n, k}^{i, p}=\theta \log _{2}\left(1+\hat{\gamma}_{e, n, k}^{i, p}\right), \forall n \in\{1,2\},
\end{aligned}
$$

where $\hat{R}_{u, k}^{i, c}$ and $\hat{R}_{e, k}^{i, c}$, respectively represent the EST and EAIR of $s_{k}^{i, c}$ corresponding to the legitimate links and to Eve's link, during the two phases. The derivation of $\hat{R}_{u, k}^{i, c}$ in (25) is presented in Appendix A and the parameter $\epsilon_{\text {out }}^{\text {max }}$ therein stands for the maximum tolerable OP. Furthermore, $\hat{R}_{n, k}^{i, p}$ in (27) (See Appendix A for proof) denotes the EST of the intended private streams corresponding to each legitimate link, while $\hat{R}_{e, n, k}^{i, p}$ represents the EAIR of the private streams corresponding to Eve's link.

Remark 3. (Secrecy Policy): Observe from (22) that the performance of Eve in detecting the private message is corrupted by the common stream since Eve was unable to detect it. This is achieved by designing the RS precoder so that even the best channel capacity that may achieved by Eve fails to support the minimum required common transmission rate, i.e. $r_{k}^{i, c} \geq \max _{\Delta \mathbf{f}_{e, k}^{i}, \mathbf{\Delta} \mathbf{n}_{n, k}^{i}} C_{e, k}^{i, c}$. In the meantime, we also expect that $r_{k}^{i, c} \leq \hat{R}_{u, k}^{i, c}$ is satisfied for ensuring that the common stream can be detected at both legitimate users.

It should be emphasized that, we assume the worst-case scenario in the sense of security, where imperfect CSIT is assumed at the legitimate transmitter and both legitimate users adjust their strategy based on the estimated channel coefficients. So, the actual total ASR between $U A V_{i}$ and each legitimate user, defined as the sum of the ASR values due to

${ }^{6}$ Note that, in case of perfect CSIT, the actual channel capacity and AIR from the Tx viewpoint are equal [29]. Whereas, under the channel uncertainties, the channel capacity cannot be achieved by Tx as the channels were not be correctly estimated. 
the common and private streams, is given by:

$$
R_{s e c, n, k}^{i, t o t} \triangleq R_{s e c, n, k}^{i, c}+R_{s e c, n, k}^{i, p}, \forall n \in\{1,2\}
$$

$R_{s e c, n, k}^{i, c} \triangleq \alpha_{n, k}^{i}\left[\hat{R}_{u, k}^{i, c}-C_{e, k}^{i, c}\right]^{+}, R_{s e c, n, k}^{i, p} \triangleq\left[\hat{R}_{n, k}^{i, p}-C_{e, n, k}^{i, p}\right]$

where we define the operation $[x]^{+} \triangleq \max (0, x)$. The weighting factors $\left.\alpha_{n, k}^{i}\right|_{n=1} ^{2} \in[0,1]$ associated with $\alpha_{1, k}^{i}+\alpha_{2, k}^{i}=1$ allow us to fine-tune the significance of the portion of each user in the overall secrecy rate enhancement. Although in [29] this coefficient is simply assumed as a constant, Here we optimize it with the aim of maximizing the ASR of the system.

Remark 4. (WCSR-CSI-errors): Under imperfect CSIT assumption, the estimated MRT beamformer $\hat{\mathbf{p}}_{z}$ no longer performs at its best and some AN is leaked towards $U_{2, k}^{i}$, as well. To alleviate this deleterious impact and guarantee a robust design against such imperfections, we will aim for maximizing the Worst-Case Secrecy Rate (WCSR), i.e. maximizing the minimum ASR over all possible CSI uncertainties.

\section{Proposed Robust WCSR Maximization}

\section{A. Problem Formulation}

To establish secrecy-rate fairness among the legitimate users, we aim for maximizing the WCSR among all legitimate users (WCSR-Fairness). It should also be remarked that, to prevent outage of legitimate links due to imperfect CSIT, transmission of common and private streams during two cooperative phases must be protected and thus, considering the following OP constraints $\forall n \in\{1,2\}$ is of vital importance:

$$
\begin{aligned}
& \mathbb{P}\left\{\hat{R}_{n, k}^{i, c(1)} \geq C_{n, k}^{i, c(1)}\right\} \leq \epsilon_{\text {out }}^{\max },(a) \\
& \mathbb{P}\left\{\hat{R}_{2, k}^{i, c(2)} \geq C_{2, k}^{i, c(2)}\right\} \leq \epsilon_{\text {out }}^{\max }(b), \\
& \mathbb{P}\left\{\hat{R}_{n, k}^{i, p} \geq C_{n, k}^{i, p}\right\} \leq \epsilon_{\text {out }}^{\max },(c) .
\end{aligned}
$$

Based on the above discussions, the optimization problem of the RS-based downlink in UAV cells is formulated as:

$$
\max _{\mathbf{P}_{k}^{i}, \theta, \mathbf{P}, \boldsymbol{\alpha}_{n, k}^{i}}\left(\min _{n \in\{1,2\}}\left(\min _{\triangle \mathbf{f}_{e, k}^{i}, \Delta \mathbf{n}_{n, k}^{i}}\left\{R_{s e c, n, k}^{i, t o t}\right\}\right)\right)
$$

s.t.

$$
\begin{array}{ll}
C_{1}: r_{k}^{i, c} \leq \hat{R}_{u, k}^{i, c}, & C_{2}: r_{k}^{i, c} \geq \max _{\triangle \mathbf{f}_{e, k}^{i}, \Delta \mathbf{n}_{n, k}^{i}} C_{e, k}^{i, c}, \\
C_{3}:(2),(30) . & C_{4}: \sum_{i=1}^{I} p_{k}^{i}\left\|\mathbf{h}_{i, k}^{M}\right\|^{2} \leq I_{k}^{i}, \forall k, \\
C_{5}: 0 \leq \alpha_{n, k}^{i} \leq 1, & C_{6}: \alpha_{1, k}^{i}+\alpha_{2, k}^{i}=1, \quad \forall i, k \\
C_{7}: 0 \leq p_{R} \leq \bar{P}_{R} \quad C_{8}: 0 \leq p_{J} \leq \bar{P}_{J} \\
C_{9}: 0 \leq \mathbf{P}^{T} \mathbf{1}_{2} \leq \bar{P}^{(2)}
\end{array}
$$

where $p_{k}^{i} \triangleq \operatorname{Tr}\left(\mathbf{P}_{k}^{i} \mathbf{P}_{k}^{i}{ }^{H}\right), \boldsymbol{\alpha}_{n, k}^{i} \triangleq\left[\alpha_{1, k}^{i}, \alpha_{2, k}^{i}\right]^{T}, \mathbf{1}_{2} \triangleq$ $[1,1]^{T}, \mathbf{P} \triangleq\left[p_{R}, p_{J}\right]^{T}$.

Based on the constraint $\left(31-C_{4}\right)$, it should be emphasized that, as the macro users have higher priority in using the spectrum, we expect that the IMCI power contaminating each macro user is kept below the maximum tolerable interference

\section{Algorithm 1 Proposed SPCA-Based Algorithm}

Input: Set the threshold value for accuracy $\left(\delta_{I}\right)$ and the maximum number of iterations $\left(N_{\max }\right)$.

Initialization: Initialize $\mathbf{x}^{[m]}$ with feasible IP and $m=0$. While $\left\{\left|t_{s e c}^{[m+1]}-t_{s e c}^{[m]}\right| \geq \delta_{I}\right.$ or $m \leq N_{\max }$ do (1) to (3):

(1).Assign the solution to $\mathbf{x}^{[m+1]}$ by solving (43).

(2). Update the slack variables based on $\mathbf{x}^{[m+1]}$.

(3). $m=m+1$.

End While,

Output: $\mathbf{P}_{k}^{i *}, \boldsymbol{\alpha}_{k}^{i *}, \theta^{*}, \mathbf{P}^{*}$.

level $I_{k}$. This is stipulated in $\left(31-C_{4}\right)$, where $\mathbf{h}_{i, k}^{M}$ represents the corresponding CSI between $U A V_{i}$ and the $M$-th macro user under $S C_{k}$. We also note that, the constraints (30) lead to a challenging optimization problem. As an alternative approach, we consider the specific scenario for these constraints in which no outage will occur at all. In other words, it is expected that the minimum capacities achieved by the legitimate links are indeed able to support the specific AIR from the Tx point of view in which the OP will become zero, i.e. $\epsilon_{\text {out }}^{\max }=0$. This can be expressed as:

$$
\begin{gathered}
\min _{\Delta \mathbf{n}_{n, k}^{i}}\left\{C_{n, k}^{i, c(1)}\right\} \geq\left.\hat{R}_{n, k}^{i, c}(1)\right|_{\epsilon_{\text {out }}^{\max }=0}, \forall n \in\{1,2\} \\
\min _{\Delta \mathbf{f}_{e, k}^{i}, \Delta \mathbf{n}_{n, k}^{i}}\left\{C_{2, k}^{i, c}(2)\right\} \geq\left.\hat{R}_{2, k}^{i, c}(2)\right|_{\epsilon_{\text {out }}^{\max }=0}, \\
\min _{\mathbf{\Delta n}_{n, k}^{i}}\left\{C_{n, k}^{i, p}\right\} \geq\left.\hat{R}_{n, k}^{i, p}\right|_{\epsilon_{\text {out }}^{\max }=0}, \forall n \in\{1,2\} .
\end{gathered}
$$

The problem (31) is still hard to solve because of the nonconvex objective function (OF) as well as the non-convex constraint set. Therefore, finding the global optimum is computationally inefficient or might even be intractable, hence finding a local optimum is more sensible in practice. To circumvent the non-convexity, we resort to using SPCA. Accordingly, the problem is approximated iteratively by a sequence of convex programs. At each iteration, the non-convex constraints are surrogated by their corresponding convex approximation counterparts.

\section{B. SPCA-based Solution}

In order to utilize SPCA, we first aim for eliminating the non-convexity by variable transformations and linearization for reformulating the problem. Then, a well-suited convex subset is constructed via SPCA that approximates the original nonconvex solution set. More explicitly, we adopt an iterative solution for dealing with the non-convexity by approximating the non-convex factor at each iteration by its first order Taylor expansion. Given this perspective, in what follows, we deal with each non-convex part independently to acquire its equivalent convex counterpart.

1) Non-Convex OF together with Constraint ( $\left.31-C_{1}\right)$ : Observing the max-min problem (31), we first aim for eliminating the inner maximizations, which are actually constituted by a pair of consecutive searches over the legitimate values of uncertainties to find the worst-case and thus can be merged 
together as $\min \left\{R_{\text {sec }, 1, k}^{i, t o t}, R_{s e c, 2, k}^{i, t o t}\right\}$. To do so, we introduce the auxiliary variable $r_{\text {sec }}$ and reformulate the OF, together with the first constraint of (31) as follows:

$$
\max _{\mathbf{P}_{k}^{i}, \theta, \mathbf{P}, \boldsymbol{\alpha}_{n, k}^{i}} r_{s e c}
$$

s.t.

$$
\begin{aligned}
C_{1}: & \alpha_{n, k}^{i}[\underbrace{\min \left\{\hat{R}_{1, k}^{i, c}, \hat{R}_{2, k}^{i, c}\right\}}_{\hat{R}_{u, k}^{i, c}}-C_{e, k}^{i, c}]^{+}+ \\
& {\left[\hat{R}_{n, k}^{i, p}-C_{e, n, k}^{i, p}\right]^{+} \geq r_{s e c} \quad \forall n \in\{1,2\} } \\
C_{2}: & \min \left\{\hat{R}_{1, k}^{i, c}, \hat{R}_{2, k}^{i, c}\right\} \geq r_{k}^{i, c} .
\end{aligned}
$$

Upon observing (33), it can be perceived that $r_{\text {sec }}$ plays the role of lower bound for $\min \left\{R_{\text {sec }, 1, k}^{i, t o t}, R_{s e c, 2, k}^{i, t o t}\right\}$ and its maximization will increase the left-side of the constraints $C_{1}$, so that it would be active at the optimum. We note that to arrive at (33), $R_{s e c, n, k}^{i, t o t}$ and $\hat{R}_{u, k}^{i, c}$ in (33- $\left.C_{1}\right)$ were respectively substituted by their definitions from (29) and (25). Furthermore, constraint $C_{2}$ arises by inserting the definition of $\hat{R}_{u, k}^{i, c}$ from (25) into (31- $\left.C_{1}\right)$.

Due to the operator $[.]^{+}$, the constraint $\left(33-C_{1}\right)$ is nonconvex. To relax the problem and to cope with non-convexity, we introduce two extra artificial constraints in (34- $\left.C_{7}\right)$, (34$C_{8}$ ) and remove this operator. To eliminate the min function in $\left(33-C_{2}\right)$ we split it into two extra constraints in $\left(34-C_{2}\right)$. Furthermore, in order to facilitate the procedure of convexifying (33), we further define two sets of new auxiliary variables $\boldsymbol{\alpha}_{p} \triangleq\left[\alpha_{p, 1}, \alpha_{p, 2}, \alpha_{1, e}, \alpha_{2, e}\right]$ and $\boldsymbol{\alpha}_{c} \triangleq\left[\alpha_{c, 1}, \alpha_{c, 2}, \alpha_{c, e}\right]$. This allows us to transform (33), $\forall n \in\{1,2\}$ into:

$$
\max _{\mathbf{P}_{k}^{i}, \boldsymbol{\alpha}_{n, k}^{i}, \boldsymbol{\alpha}_{p}, \boldsymbol{\alpha}_{c}} r_{s e c}
$$

s.t.

$$
\begin{aligned}
& C_{1}: \alpha_{n, k}^{i}\left(\gamma_{c}-\alpha_{c, e}\right)+\alpha_{p, n}-\alpha_{n, e} \geq r_{s e c}, \\
& C_{2}: \\
& \gamma_{c} \geq r_{k}^{i, c}, \alpha_{c, n} \geq \gamma_{c}, \quad C_{3}: \hat{R}_{n, k}^{i, p} \geq \alpha_{p, n}, \\
& C_{4}: \hat{R}_{n, k}^{i, c} \geq \alpha_{c, n}, \quad C_{5}: C_{e, n, k}^{i, p} \leq \alpha_{n, e}, \\
& C_{6}: C_{e, k}^{i, c} \leq \alpha_{c, e}, \quad C_{7}: \alpha_{c, n} \geq \alpha_{c, e}, \alpha_{p, n} \geq \alpha_{n, e},
\end{aligned}
$$

Based on the above discussion, $\alpha_{p, n}$ and $\alpha_{c, n}$ can respectively represent the lower-bound of $\hat{R}_{n, k}^{i, p}$ and $\hat{R}_{n, k}^{i, c}$, whilst $\alpha_{n, e}$ and $\alpha_{c, e}$ stand as the upper-bounds of $C_{e, n, k}^{i, p}$ and $C_{e, k}^{i, c}$, respectively. Increasing the lower-bound values and simultaneously reducing the upper-bounds will boost the left-side of the constraints, which is needed here, so that the constraints (34- $C_{1}: C_{6}$ ) would be active at the optimum.

Despite this linearization, it can be seen by invoking the definitions of $\hat{R}_{n, k}^{i, p}, \hat{R}_{e, n, k}^{i, p}, C_{e, n, k}^{i, p}, C_{e, k}^{i, c}$ that the constraints (34- $\left.C_{1}, C_{3}: C_{6}\right)$ are still non-convex. To handle the non-convexity of these constraints we construct a suitable inner convex subset for approximating the nonconvex feasible solution set. Along this line, we first approximate $\left(34-C_{1}\right)$ by its linear Taylor expansion to obtain $\Theta^{[m]}\left(\alpha_{n, k}^{i}, \gamma_{c}\right)-\bar{\Theta}^{[m]}\left(\alpha_{n, k}^{i}, \alpha_{c, e}\right)+\alpha_{p, n}-\alpha_{n, e} \geq t_{s e c}$, where we have defined $\Theta^{[m]}(x, y) \triangleq \frac{1}{2}\left(x^{[m]}+\right.$ $\left.y^{[m]}\right)(x+y)-\frac{1}{4}\left(x^{[m]}+y^{[m]}\right)^{2}-\frac{1}{4}(x-y)^{2}, \quad$ and $\bar{\Theta}^{[m]}(x, y) \triangleq \frac{1}{4}(x+y)^{2}+\frac{1}{4}\left(x^{[m]}-y^{[m]}\right)^{2}-\frac{1}{2}\left(x^{[m]}-\right.$ $\left.y^{[m]}\right)(x-y)$ for the linear approximation of the terms, which are the product of two variables.

Next, in order to handle the constraints $\left(34-C_{3}: C_{6}\right)$, we first define four sets of new auxiliary variables $\boldsymbol{\beta}_{c}=$ $\left[\beta_{c, 1}^{(1)}, \beta_{c, 2}^{(1)}, \beta_{c, 2}^{(2)}, \beta_{c, e}^{(1)}, \beta_{c, e}^{(2)}\right], \boldsymbol{\rho}_{c}=\left[\rho_{c, 1}^{(1)}, \rho_{c, 2}^{(1)}, \rho_{c, 2}^{(2)}, \rho_{c, e}^{(1)}, \rho_{c, e}^{(2)}\right]$, $\boldsymbol{\beta}_{p}=\left[\beta_{p, 1}, \beta_{p, 2}, \beta_{1, e}, \beta_{2, e}\right], \boldsymbol{\rho}_{p}=\left[\rho_{p, 1}, \rho_{p, 2}, \rho_{1, e}, \rho_{2, e}\right]$ and exploit the following lemmas.

Lemma 5. The constraint (34- $\left.C_{3}\right), \forall n, j \in\{1,2\}$ is approximated by its convex set counterpart as follows:

$$
\left\{\begin{array}{l}
(I): \beta_{p, n} \geq \alpha_{p, n} \\
(I I):\left|\left(\hat{\mathbf{h}}_{n, k}^{i}\right)^{H} \mathbf{p}_{j, k}^{i, p}\right|^{2}+p_{M, k}\left\|\mathbf{c}_{n, k}^{i}\right\|^{2}+\delta^{2}- \\
\Psi^{[m]}\left(\mathbf{p}_{n, k}^{i, p}, \rho_{p, n} ; \hat{\mathbf{h}}_{n, k}^{i}\right) \leq 0, n \neq j \\
(I I I): 1+\rho_{p, n}-2^{\beta_{p, n}} \geq 0,
\end{array}\right.
$$

where the operators are defined as $\Psi^{[m]}(\mathbf{u}, x ; \mathbf{h}) \triangleq$ $\frac{2 \Re e\left\{\left(\mathbf{u}^{[m]}\right)^{H} \mathbf{h h}^{H} \mathbf{u}\right\}}{x^{[m]}}-\frac{\left|\mathbf{h}^{H} \mathbf{u}^{[m]}\right|^{2} x}{\left(x^{[m]}\right)^{2}}$.

Proof: see Appendix B.

Lemma 6. The affine approximation of constraint (34- $\left.C_{4}\right)$, $\forall n \in\{1,2\}$ is given by:

$$
\left\{\begin{array}{l}
(I): \Theta^{[m]}\left(\theta, \beta_{c, 1}^{(1)}\right) \geq \alpha_{c, 1}, \\
(I I): \Theta^{[m]}\left(\theta, \beta_{c, 2}^{(1)}\right)+\Theta^{[m]}\left(1-\theta, \beta_{c, 2}^{(2)}\right) \geq \alpha_{c, 2}, \\
(I I I): \beta_{c, n} \geq \alpha_{c, n}, \\
(I V):\left|\left(\hat{\mathbf{h}}_{n, k}^{i}\right)^{H} \mathbf{p}_{1, k}^{i, p}\right|^{2}+\left|\left(\hat{\mathbf{h}}_{n, k}^{i}\right)^{H} \mathbf{p}_{2, k}^{i, p}\right|^{2}+\delta^{2} \\
+p_{M, k}|| \mathbf{c}_{n, k}^{i} \|^{2}-\Psi^{[m]}\left(\mathbf{p}_{k}^{i, c}, \rho_{c, n}^{(1)} ; \hat{\mathbf{h}}_{n, k}^{i}\right) \leq 0 \\
(V): p_{j}\left|\left(\hat{\mathbf{h}}_{2, k}^{i}\right)^{H} \hat{\mathbf{p}}_{z}\right|^{2}+p_{M, k}\left\|\mathbf{c}_{n, k}^{i}\right\|^{2}+\sigma^{2}- \\
\Psi(m]\left(\delta_{R}, \rho_{c, 2}^{(2)} ; \hat{f}_{2, k}^{i}\right) \leq 0 \\
(V I): 1+\rho_{c, 1}^{(1)}-2^{\beta_{c, 1}^{(1)}} \geq 0 \\
(V I I): 1+\rho_{c, 2}^{(j)}-2^{\beta_{c, 2}^{(j)}} \geq 0
\end{array}\right.
$$

where $p_{R} \triangleq \delta_{R}^{2}$.

Proof: Following the same approach we presented in Appendix B, Lemma 6 can be proved.

Lemma 7. The affine approximation of constraint (34- $\left.C_{5}\right)$, $\forall n, j \in\{1,2\}$ is given by:

$$
\left\{\begin{array}{l}
(I): \bar{\Theta}^{[m]}\left(\theta, \beta_{n, e}\right) \leq \alpha_{n, e}, \\
(I I): \Omega^{[m]}\left(\mathbf{p}_{k}^{i, c}, \mathbf{p}_{j, k}^{i, p} ; \mathbf{h}_{e, k}^{(1)}\right)+p_{M, k}\left\|\mathbf{w}_{e, k}^{(1)}{ }^{H} \mathbf{C}_{e, k}^{M, i}\right\|^{2} \\
+\delta^{2}-\frac{\left|\mathbf{h}_{e, k}^{(1)} \mathbf{p}_{n, k}^{i, p}\right|^{2}}{\rho_{n, e}} \geq 0, n \neq j \\
(I I I): 1+\rho_{n, e}-\Gamma^{[m]}\left(\beta_{n, e}\right) \leq 0
\end{array}\right.
$$

and the operators used therein are defined as $\Omega^{[m]}(\mathbf{u}, \mathbf{v} ; \mathbf{h}) \triangleq \Psi^{[m]}(\mathbf{u}, 1 ; \mathbf{h})-\Psi^{[m]}(\mathbf{v}, 1 ; \mathbf{h})$, 


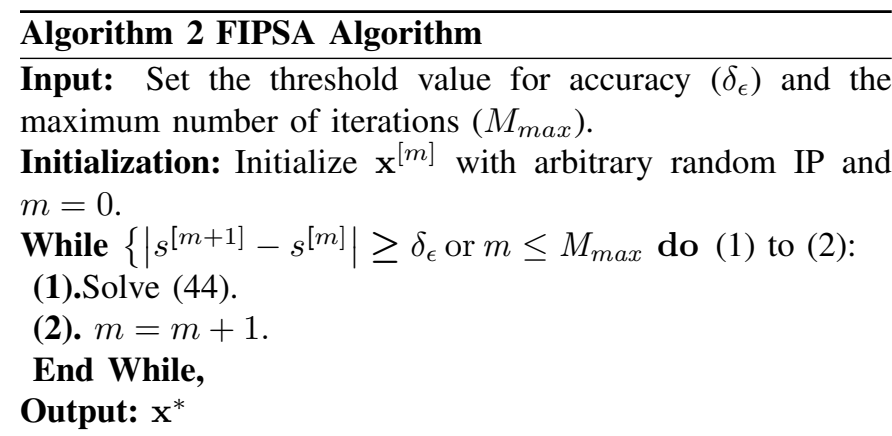

$\Gamma^{[m]}(x) \triangleq 2^{x^{[m]}}\left[1+\ln (2)\left(x-x^{[m]}\right)\right]$.

Proof: see Appendix C.

Lemma 8. The affine approximation of constraint (34- $C_{6}$ ), $\forall n \in\{1,2\}$ is given by:

$$
\left\{\begin{array}{l}
(I): \bar{\Theta}^{[m]}\left(\theta, \beta_{c, e}^{(1)}\right)+\bar{\Theta}^{[m]}\left(1-\theta, \beta_{c, e}^{(2)}\right) \leq \alpha_{c, e}, \\
(I I): \Omega^{[m]}\left(\mathbf{p}_{1, k}^{i, p}, \mathbf{p}_{2, k}^{i, p} ; \mathbf{h}_{e, k}^{(1)}\right)+p_{M, k}\left\|\mathbf{w}_{e, k}^{(1)} \mathbf{C}_{e, k}^{M, i}\right\|^{2} \\
+\delta^{2}-\frac{\left|\mathbf{h}_{e, k}^{(1)} \mathbf{p}_{k}^{i, c}\right|^{2}}{\rho_{c, e}^{(1)}} \geq 0, \\
(I I I): p_{M, k}\left\|\mathbf{w}_{e, k}^{(2)}{ }^{H} \mathbf{C}_{e, k}^{M, i}\right\|^{2}+\delta^{2}+p_{j}\left|\mathbf{w}_{e, k}^{(2)^{H}} \hat{\mathbf{p}}_{z}\right|^{2}-\frac{\delta_{R}^{2}}{\rho_{c, e}^{(2)}} \geq 0 \\
(V): 1+\rho_{c, e}^{(j)}-\Gamma^{[m]}\left(\beta_{c, e}^{(j)}\right) \leq 0, \forall j \in\{1,2\} .
\end{array}\right.
$$

Proof: Following the same approach as presented in Appendix B, Lemma 8 can be proved.

2) Non-convex constraint $\left(31-C_{2}\right)$ : To handle the nonconvexity of (31- $\left.C_{2}\right)$, we first introduce the new auxiliary variables of $\pi_{c, e} \triangleq\left[u_{1, c, e}, u_{1, c, e}, v_{c, e}, x_{c, e}, y_{c, e}, d_{c, e}, f_{c, e}\right]$ and formulate Lemma 9, as follows:

Lemma 9. The affine approximation of constraint $\left(31-C_{2}\right)$ is given by:

$$
\left\{\begin{array}{l}
(I): \alpha_{c, e} \leq r_{k}^{i, c}, \\
(I I): \sum u_{n, c, e}^{2}+p_{M, k}\left\|\left(\hat{\mathbf{w}}_{e, k}^{(1)}\right)^{H} \mathbf{C}_{e, k}^{M, i}\right\|^{2}+\delta^{2} \geq d_{c, e} \\
(I I I): \frac{x_{c, e}^{2}}{d_{c, e}} \leq \rho_{c, e}^{(1)} \\
(I V): v_{c, e}+p_{M, k}\left\|\left(\hat{\mathbf{w}}_{e, k}^{(2)}\right)^{H} \mathbf{C}_{e, k}^{M, i}\right\|^{2}+\delta^{2} \geq f_{c, e}, \\
(V): \frac{\delta_{R}^{2}}{f_{c, e}} \leq \rho_{c, e}^{(2)} \\
(V I):\left|\left(\hat{\mathbf{h}}_{e, k}^{(1)}\right)^{H} \mathbf{p}_{k}^{i, c}\right|+\sqrt{N_{e} N_{t}} \xi\left\|\mathbf{p}_{k}^{i, c}\right\|_{2} \leq x_{c, e} \\
(V I I): \operatorname{Tr}\left[\left(\mathbf{Q}_{e, k}^{(1)}-\mu_{e} \mathbf{I}\right) \mathbf{P}_{n, k}^{i, p}\right] \geq u_{n, c, e} \\
(V I I I): p_{j} \operatorname{Tr}\left[\left(\mathbf{Q}_{e, k}^{(2)}-\mu_{e} \mathbf{I}\right) \hat{\mathbf{P}}_{z}\right] \geq v_{c, e}
\end{array}\right.
$$

where we have $\mathbf{Q}_{e, k}^{(1)} \triangleq \hat{\mathbf{h}}_{e, k}^{(1)}\left(\hat{\mathbf{h}}_{e, k}^{(1)}\right)^{H}, \mathbf{Q}_{e, k}^{(2)} \triangleq \hat{\mathbf{w}}_{e, k}^{(2)}\left(\hat{\mathbf{w}}_{e, k}^{(2)}\right)^{H}$, $\hat{\mathbf{h}}_{e, k}^{(n)} \triangleq\left(\hat{\mathbf{H}}_{e, k}^{i}\right)^{H} \hat{\mathbf{w}}_{e, k}^{(n)}, \mathbf{P}_{n, k}^{i, p} \triangleq \mathbf{p}_{n, k}^{i, p}\left(\mathbf{p}_{n, k}^{i, p}\right)^{H}$, and $\mu_{e}=$ $N_{e} N_{t} \zeta^{2}+2 \sqrt{N_{e} N_{t}} \zeta\left\|\hat{\mathbf{H}}_{e, k}^{i}\right\|_{2}$.

Proof: See Appendix D .
3) Non-convex constraint $\left(31-C_{3}\right)$ : Similar to the above statements, to handle the non-convexity of $\left(31-C_{3}\right)$, we have introduced new auxiliary constraints, corresponding to each constraint. In this regard, we introduce new collections of auxiliary variables $\boldsymbol{\pi}_{p, n} \triangleq\left[\gamma_{p, n}, t_{p, n}, \omega_{p, j}, v_{j, p, n}\right]$ and $\boldsymbol{\pi}_{c, n}^{(1)} \triangleq$ $\left[f_{c, n}, g_{c, n}, x_{c, n}^{(1)}, d_{c, n}^{(1)}, \gamma_{c, n}^{(1)}\right], \boldsymbol{\pi}_{c, n}^{(1)} \triangleq\left[x_{c, n}^{(2)}, d_{c, n}^{(2)}, \gamma_{c, n}^{(2)}\right]$ that are used in the following lemmas.

Lemma 10. The affine approximation of constraint (30-c), $\forall n, j \in\{1,2\}, n \neq j$ is given by:

$$
\left\{\begin{array}{l}
(I): \frac{\left|\left(\hat{\mathbf{h}}_{n, k}^{i}\right)^{H} \mathbf{p}_{n, k}^{i, p}\right|^{2}}{\gamma_{p, n}}-\Psi^{[m]}\left(\mathbf{p}_{j, k}^{i, p}, 1 ; \hat{\mathbf{h}}_{n, k}^{i}\right)- \\
p_{M, k}|| \mathbf{c}_{n, k}^{i} \|^{2}-\delta^{2} \leq 0, \\
(I I): \gamma_{p, n}-\mathcal{T}^{[m]}\left(t_{p, n}, w_{p, j}\right) \leq 0, \\
(I I I): t_{p, n}+\kappa_{n} \operatorname{Tr}\left[\mathbf{P}_{n, k}^{i, p}\right]-\Psi^{[m]}\left(\mathbf{p}_{n, k}^{i, p}, 1 ; \hat{\mathbf{h}}_{n, k}^{i}\right) \leq 0, \\
(I V): v_{j, p, n}-w_{p, j}+p_{M, k}\left\|\mathbf{c}_{n, k}^{i}\right\|^{2}+\delta^{2} \leq 0, \\
(V): \operatorname{Tr}\left[\left(\hat{\mathbf{H}}_{n, k}+\kappa_{n} \mathbf{I}\right) \mathbf{P}_{j, k}^{i, p}\right]-v_{j, p, n} \leq 0,
\end{array}\right.
$$

where $\hat{\mathbf{H}}_{n, k} \triangleq \hat{\mathbf{h}}_{n, k}^{i}\left(\hat{\mathbf{h}}_{n, k}^{i}\right)^{H}, \mathbf{P}_{j, k}^{i, p} \triangleq \mathbf{p}_{j, k}^{i, p} \mathbf{p}_{j, k}^{i, p^{H}}, \kappa_{n}=$ $N_{t} \zeta^{2}+2 \sqrt{N_{t}} \zeta\left\|\hat{\mathbf{h}}_{n, k}^{i}\right\|_{2}$, and $\mathcal{T}^{[m]}(x, y) \triangleq \frac{x}{y^{[m]}}-\frac{x^{[m]}}{y^{[m]^{2}}}(y-$ $\left.y^{[m]}\right)$.

Proof: See Appendix E.

Lemma 11. The affine approximation of constraint (30-b), $\forall n, j \in\{1,2\}, n \neq j$ is given by:

$$
\left\{\begin{array}{l}
(I): \frac{\left|\left(\hat{\mathbf{h}}_{n, k}^{i}\right)^{H} \mathbf{p}_{n, k}^{i, c}\right|^{2}}{\gamma_{c, n}}-\Omega^{[m]}\left(\mathbf{p}_{1, k}^{i, p}, \mathbf{p}_{2, k}^{i, p} ; \hat{\mathbf{h}}_{n, k}^{i}\right)- \\
\quad p_{M, k}\left\|\mathbf{c}_{n, k}^{i}\right\|^{2}-\delta^{2} \leq 0, \\
(I I): \gamma_{c, n}^{(1)}-\mathcal{T}^{[m]}\left(x_{c, n}^{(1)}, d_{c, n}^{(1)}\right) \leq 0, \\
(I I I): x_{c, n}^{(1)}+\kappa_{n} T r\left[\mathbf{P}_{k}^{i, c}\right]-\Psi^{[m]}\left(\mathbf{p}_{k}^{i, c}, 1 ; \hat{\mathbf{h}}_{n, k}^{i}\right) \leq 0 \\
(V I): f_{c, n}^{2}+g_{c, n}^{2}-d_{c, n}^{(1)}+p_{M, k}\left\|\mathbf{c}_{n, k}^{i}\right\|^{2}+\delta^{2} \leq 0, \\
(V):\left|\left(\hat{\mathbf{h}}_{n, k}^{i}\right)^{H} \mathbf{p}_{1, k}^{i, p}\right|+\sqrt{N_{t}} \zeta\left\|\mathbf{p}_{1, k}^{i, p}\right\|_{2}-f_{c, n} \leq 0, \\
(V I):\left|\left(\hat{\mathbf{h}}_{n, k}^{i}\right)^{H} \mathbf{p}_{2, k}^{i, p}\right|+\sqrt{N_{t}} \zeta\left\|\mathbf{p}_{2, k}^{i, p}\right\|_{2}-g_{c, n} \leq 0 .
\end{array}\right.
$$

Proof: Following the same approach we presented in Appendix E, Lemma 11 can be proved.

Lemma 12. Similarly, the affine approximation of constraint ((31)- $a), \forall n, j \in\{1,2\}, n \neq j$ is given by:

$$
\left\{\begin{array}{l}
(I): p_{j}\left|\left(\hat{\mathbf{h}}_{2, k}^{i}\right)^{H} \hat{\mathbf{p}}_{z}\right|^{2}+p_{M, k}\left\|\mathbf{c}_{2, k}^{i}\right\|^{2}+\delta^{2}- \\
\Omega^{[m]}\left(\delta_{R}, \gamma_{c, 2}^{(2)} ; \hat{f}_{2, k}^{i}\right) \leq 0, \\
(I I): \gamma_{c, 2}^{(2)}-\mathcal{T}^{[m]}\left(x_{c, 2}^{(2)}, d_{c, 2}^{(2)}\right) \leq 0, \\
(I I I): x_{c, 2}^{(2)}-\left(\left|\hat{f}_{2, k}^{i}\right|^{2}-\varrho\right) \Lambda^{[m]}\left(\delta_{R}\right) \leq 0, \\
(I V): v_{j, p, n}-w_{p, j}+p_{M, k}\left\|\mathbf{c}_{n, k}^{i}\right\|^{2}+\delta^{2} \leq 0, \\
(V): p_{j} \operatorname{Tr}\left[\left(\hat{\mathbf{H}}_{2, k}+\kappa_{2} \mathbf{I}\right) \hat{\mathbf{P}}_{z}\right]-d_{c, 2}^{(2)}+p_{M, k}\left\|\mathbf{c}_{2, k}^{i}\right\|^{2}+ \\
\quad \delta^{2} \leq 0,
\end{array}\right.
$$


where $\varrho=\xi^{2}+2 \xi\left|\hat{f}_{2, k}^{i}\right|^{2}, \quad \Lambda^{[m]}(x)=\left(x^{[m]}\right)^{2}+$ $2 x^{[m]}\left(x-x^{[m]}\right)$.

Proof: Following the same approach we presented in Appendix E, Lemma 12 can be proved.

\section{Overall Proposed SPCA-BASEd Algorithm}

Given the above approximations, the proposed SPCA-based procedure is summarized in Algorithm 1, where the convex approximation program (43) is solved at the $m$-th iteration using standard numerical optimization packages, like CVX [42]. If there exists feasible initial points for (43), the feasible set defined by the constraints of (43) and the consequently resultant solutions definitely fall within the original feasible set defined by (31- $\left.C_{1}: C_{9}\right)$. The procedure continues, unless the stopping criterion is satisfied or the pre-determined number of iterations is exhausted:

$$
\max _{\mathbf{x}} r_{s e c}
$$

s.t.

$$
\begin{aligned}
& \left(34-\left(C_{1}: C_{2}\right)\right),\left(34-C_{7}\right), \\
& (35):(42),\left(32-\left(C_{4}: C_{6}\right)\right), \varpi \succeq 0,
\end{aligned}
$$

where $\varpi \triangleq\left[\gamma_{c}, \boldsymbol{\alpha}_{p}, \boldsymbol{\alpha}_{c}, \boldsymbol{\beta}_{c}, \boldsymbol{\beta}_{p}, \boldsymbol{\rho}_{c}, \boldsymbol{\rho}_{p}, \boldsymbol{\pi}_{c, e}, \boldsymbol{\pi}_{p, n}, \boldsymbol{\pi}_{c, n}\right]$ and $\mathbf{x} \triangleq\left[\mathbf{P}_{k}^{i}, \boldsymbol{\alpha}_{k}^{i}, \varpi\right]$. Note that, if Algorithm 1 is initialized with random points, it may fail at the very beginning, because of infeasibility. To circumvent this issue, we now conceive a Feasible Initial Point Search Algorithm (FIPSA) in Algorithm 2. In this regard, we aim for minimizing an infeasibility indicator parameter $s>0$, to flag up any violation of the constraints of (43). Hence we have to rewrite all the 16 constraints of problem (37) in the form of $\left.\mathscr{G}_{i}(\mathbf{x})\right|_{i=1} ^{16} \leq s$, where $\mathscr{G}_{i}(\mathbf{x})$ stands for the reshaped format of the $i$-th constraint and all the terms at the left-side of the less than or equal to zero. Then we reformulate the feasibility problem as follows:

$$
\min _{\mathbf{x}} s
$$

s.t. $\left.\quad \mathscr{G}_{i}(\mathbf{x})\right|_{i=1} ^{16} \leq s$.

This approach has been previously proposed in [7] and [34] as a low-complexity scheme for finding the feasible initial points. Overall, the proposed FIPSA Algorithm 2 runs at the first step and then the Initial Points (IP)s calculated are fed to Algorithm 1. Algorithm 2 commences with random IPs and the algorithm is halted if either the stopping criterion is satisfied or the maximum number of affordable iterations is reached. We remark that, if at the $m$-th iteration the current OF $s$ is zero, the algorithm stops even though the stopping criterion is not satisfied. Before starting communications, based on the instantaneous CSIs and QoS of mobile-users, which are collected by the macro-BS, the locations of the hovering UAVs are determined through backhaul links for each specific time-frame. During this period of time, the proposed algorithm is executed and both the RS-precoder as well as the other resources are optimized for each UAV-cell based on the instantaneous CSIs. After completing each time-frame, the BS relocates the UAVs in accordance with the available knowledge. Each UAV prefers to exchange its location with its neighboring UAVs. The process of relocating the UAVs after

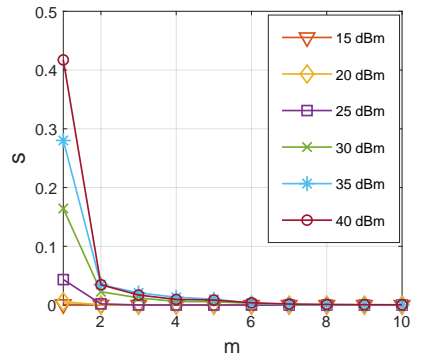

Fig. 4. Evaluating the convergence behavior of the FIPSA method versus the number of iterations for different values of $\bar{P}_{U A V}^{i}$.

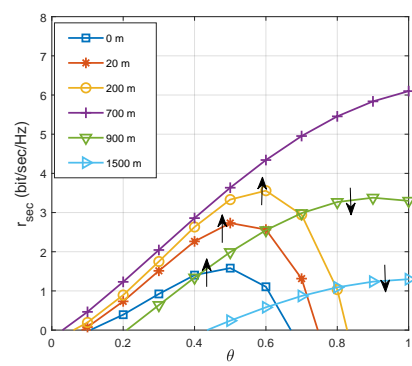

Fig. 6. Worst-case secrecy rate achieved versus the time slot sharing $\theta$ for different values of $H_{i}$.

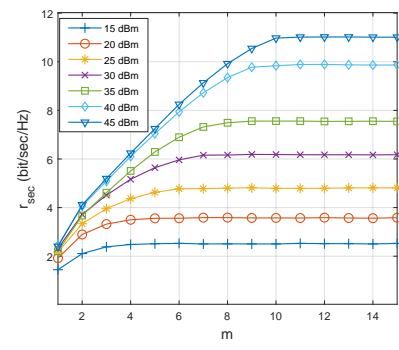

Fig. 5. Worst-case secrecy rate rate achieved by the proposed algorithm versus the number of iterations for different values of $\bar{P}_{U A V}^{i}$

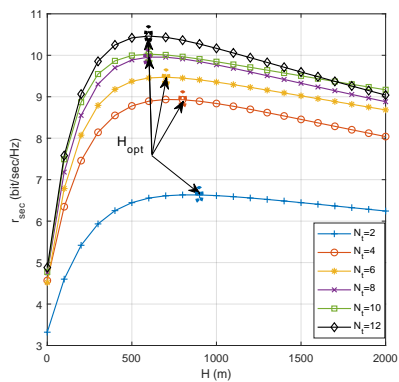

Fig. 7. Worst-case secrecy rate achieved at the UAV altitude $H_{i}$ for different number of TAs $N_{t}$. each time frame, is reminiscent of the hand-over procedure of cellular networks, but these mobility issues are beyond the scope of the current paper.

\section{Simulation Results}

In this section, we present numerical results for characterizing our proposed framework. Our simulation settings are based on the following adjustment unless otherwise stated. The BS is arranged at the center point with $R_{B S}=1000 \mathrm{~m}$ for the macrocell radius, where $N_{M}=5$ macro users are randomly distributed around the BS for receiving downlink signals from it. In the scenario investigated several UAVs randomly move over the users and hover to provide communication services. Explicitly, $I=4 \mathrm{UAVs}$ are randomly distributed within the macro cell and they have a coverage radius of $R_{U A V}=350 \mathrm{~m}$ and altitude of $H_{i}=150 \mathrm{~m}$. Furthermore, $N$ legitimate users request services in each UAV cell. Each UAV has a minimum distance constraint from the ground BS to ensure that the UAV can serve users also at the edge of the macrocell with $K=10$ sub-channels. Furthermore, enough users are randomly located within the coverage of each UAV, two legitimate user per each sub-channel $S C_{k}$. The total number of UAV users in the simulation ranges from 8 to 20 . The minimum distance between the $\mathrm{BS}$ and UAV cells is $50 \mathrm{~m}$ on a horizontal plane. In contrast to the channel model spanning from the macro BS to users, the path loss model in theUAV network includes both LOS and NLOS links associated with the path-loss exponents $\mathcal{L}_{n, k}^{i}=2$ and $\mathcal{N}_{n, k}^{i}=3.5$, respectively. We assume that the channel gain 


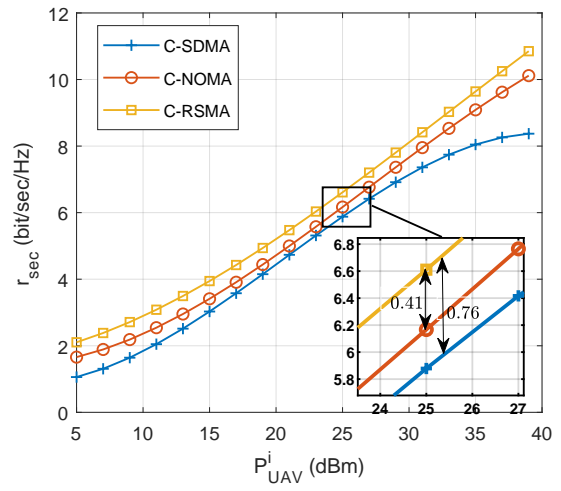

Fig. 8. Worst-case secrecy rate versus $\bar{P}_{U A V}^{i}$ for C-RSMA, CSDMA and C-NOMA.

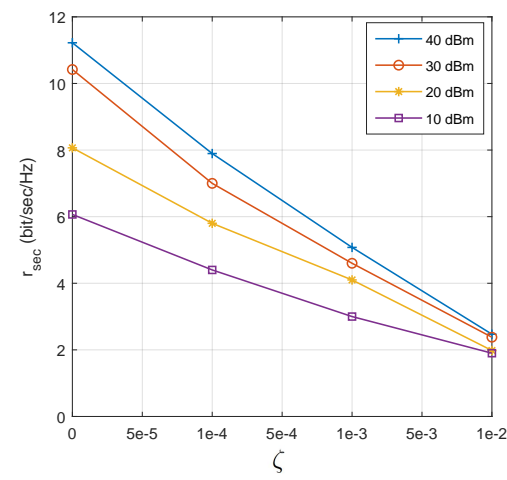

Fig. 9. Worst-case secrecy rate versus $\zeta$ parameterized by $\bar{P}_{U A V}^{i}$.

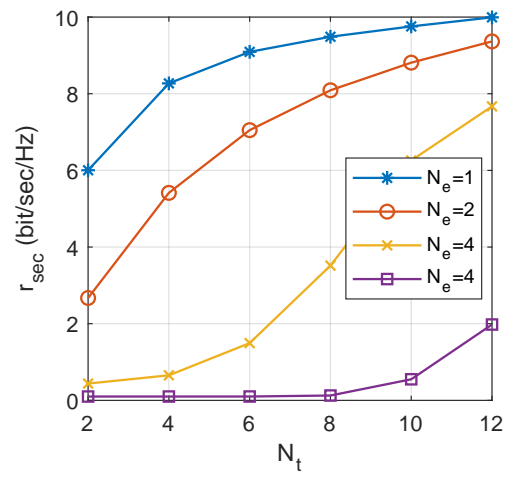

Fig. 10. Worst-case secrecy rate versus $N_{t}$ for different values of $N_{e}$. of $U_{1, k}^{i}$ is stronger than that of $U_{2, k}^{i}$. The simulation results are averaged over 500 realizations of the proposed scheme. Moreover, we set $N_{\max }=M_{\max }=20$ and the maximum tolerable threshold value is set to $\delta_{I}=10^{-2}$. The power of the macro BS is $p_{M, k}=20 \mathrm{dBm}$ for each sub-carrier. A channel estimation error with variance of $\zeta=10^{-3}$ is assumed and the additive noise at the receiver is considered to have a normalized power of $\delta^{2}=0 \mathrm{dBm}$, and the minimum required transmission rate of the common stream is $r_{k}^{i, c}=1 \mathrm{Bit} / \mathrm{sec} / \mathrm{Hz}$.

Fig. 4 depicts the average convergence of the FIPSA algorithm using the OF value of (44) versus the number of iterations for different values of UAV transmission power $\bar{P}_{U A V}^{i}$. As observed, the average convergence of the FIPSA algorithm is fast, converging at a maximum of five iterations. Interestingly, upon increasing the transmission power the algorithm's convergence rate decreases. This arises from the fact that increasing the transmission power results in higher information leakage to Eve and thus more iterations are required to meet the security constraints of (44).

The average convergence speed of the proposed SPCAbased solution of the problem in (31) is shown in Fig. 5. The results show the convergence of Algorithm 1 at a maximum of 10 iterations. Similar to the convergence behavior of FIPSA, upon increasing the transmit power the algorithm's convergence rate decreases.

Fig 5 shows the worst-case average secrecy rate achieved by the proposed framework versus the time slot sharing parameter $\theta$. In this experiment we aim for observing the impact of UAV altitude $H_{i}$ and $\theta$ on the achievable secrecy rate. Interestingly, the $\mathrm{OF}$ versus $\theta$ is concave and there is an optimum $\theta^{*}$ at which the secrecy rate achieved will be maximized. This figure also indicates that the secrecy rate critically depends on the altitude of the UAV and at the optimum $\left(H_{i}^{*}\right)$ we have $\theta^{*}=1$. This indicates that at the optimal altitude the CRS framework is the non-cooperative RS, where no cooperative phase is required. This is because, owing to the LoS links, the quality of the common signal received at cell-edge user is good enough at the optimal altitude. Hence no cooperation is needed for relaying the common stream. Observe in Fig. 6 that, by increasing the altitude and getting away from the optimal altitude, the distances of LoS links to both users will be almost identical and the secrecy rate is reduced due to the increased path-loss. By contrast, upon reducing the UAV altitude from the optimal ones, $\theta^{*}$ is deceased as well because of the emergence of fading. Hence the common signal received by the cell-edge user is corrupted and reliance on the cooperative phase becomes inevitable.

To reveal the joint impact of the UAV altitude $H_{i}$ and number of TAs $N_{t}$ we have generated Fig. 7. On one hand, as expected, the secrecy rate is boosted by increasing the number of TAs at the UAV, owing to the increased DoF provided for beamforming. On the other hand, as the number of TAs becomes high, the best UAV altitude is decreased, because the extra TAs increase the DoF, hence increasing the LOS probability towards the legitimate users even at low UAV altitudes.

As mentioned in [27], Cooperative RSMA (C-RSMA) encompasses both C-NOMA and C-SDMA as special cases. Thus, upon considering dynamic time slot sharing $0<\theta<1$, if no power is allocated to $\mathbf{P}_{2, k}^{i, p}$ together with encoding the entire private message of the second user into $s_{k}^{i, c}$, a CNOMA scheme is obtained. By contrast, we arrive at C-SDMA by switching off the common stream bearing part of the transmitted signal. Given this perspective, it can be explicitly observed in Fig. 8 that the proposed strategy outperforms both its C-NOMA and C-SDMA counterparts.

For characterizing the robustness of our proposed framework against imperfect CSIT, we have produced Fig. 9 in which the average worst-case secrecy rate is depicted versus the CSIT estimation error $\zeta$. This experiment was performed for various transmit power budgets $\bar{P}_{U A V}^{i}$. Observe that regardless of the available power budget the proposed robust framework achieves a similar secrecy rate at $\zeta=10^{-2}$. By contrast, at a low CSI estimation error of $\zeta=10^{-4}$ a power budget of $40 \mathrm{dBm}$ approximately doubles the secrecy rate.

Finally, Fig. 10 illustrates the WCSR versus the number of transmit antennas for different values of receive antennas at Eve. This figure indicates that increasing the number of transmit antennas at the UAV enhances system's performance, which is an explicit benefit of the increased DoF provided by the UAV-Tx. On the other hand, upon increasing the 
number of the receive antennas at Eve, the system's secrecy performance is degraded due to Eve's improved ability to eavesdrop and infer from common message. Interestingly, our proposed scheme still shows considerable robustness against a multiple antenna-aided Eve, hence we can achieve non-zero WCSR.

\section{CONCLUSIONS}

In this paper, we conceived robust and secure resource allocation algorithms for UAV-aided MISO-RS HetNets under imperfect CSIT for both the legitimate users and Eve, which was based on the worst-case CSI uncertainty channel model. Our CRS optimization scheme was formulated as a nonconvex robust worst-case secrecy rate maximization problem, where the common message was also used for drowning out Eve without any extra transmit power consumption. The objective of the proposed robust design is to maximize the WCSR subject to both link outage and power budget constraints. The resultant non-convex optimization problem was solved using the SPCA method. In order to circumvent any potential infeasibility, we have also proposed an iterative initialization algorithm. Our numerical results have shown the superiority of the proposed framework over both C-NOMA and C-SDMA. More importantly, our solution guarantees the security of the private messages, which is not possible for its NOMA and SDMA counterparts. In our future research we would like to consider sophisticated eavesdroppers having both an arbitrarily high number of antennas [10] as well as the impact of mobility [45].

\section{ACKNOWLEDGMENT}

L. Hanzo would like to acknowledge the financial support of the Engineering and Physical Sciences Research Council projects EP/P034284/1 and EP/P003990/1 (COALESCE) as well as of the European Research Council's Advanced Fellow Grant QuantCom (Grant No. 789028).

\section{APPENDIX A}

PROOF OF (25) AND (27)

To proceed with this proof, we first note that while the transmission rate of the common stream attained by $U_{1, k}^{i}$ relies on the first phase, $U_{2, k}^{i}$ incorporates the achievable rates at both phases, i.e. $\hat{R}_{2, k}^{i, c}=\hat{R}_{2, k}^{i, c^{(2)}}+\hat{R}_{1, k}^{i, c^{(1)}}$. So, the EST of common stream related to the legitimate users, denoted by $E S T_{n, k}^{i, c}$, can be expressed as:

$$
E S T_{n, k}^{i, c} \triangleq \hat{R}_{n, k}^{i, c}(\underbrace{1-\mathbb{P}\left\{\hat{R}_{n, k}^{i, c} \geq C_{n, k}^{i, c}\right\}}_{N O P}), \quad(A-1)
$$

$\forall n \in\{1,2\}$. Considering that the OP is bounded by $\epsilon_{\text {out }}^{\max }$, i.e. $\mathbb{P}\left\{\hat{R}_{n, k}^{i, c} \geq C_{n, k}^{i, c}\right\} \leq \epsilon_{\text {out }}^{\max }$, together with the fact that $\hat{R}_{u, k}^{i, c}$ is limited by the worst-case achievable EST among the legitimate users, the transmission rate of the common stream attained by the legitimate users from the viewpoint of the $U A V_{i}$, is formulated as follows:

$$
\begin{aligned}
& \hat{R}_{u, k}^{i, c} \triangleq \min _{n \in\{1,2\}}\left\{E S T_{n, k}^{i, c}\right\}=\min \left\{\left(1-\mathbb{P}\left\{\hat{R}_{1, k}^{i, c} \geq C_{1, k}^{i, c}\right\}\right)\right. \\
& \left.\hat{R}_{1, k}^{i, c},\left(1-\mathbb{P}\left\{\hat{R}_{2, k}^{i, c} \geq C_{2, k}^{i, c}\right\}\right) \hat{R}_{2, k}^{i, c}\right\} \\
& =\left(1-\epsilon_{\text {out }}^{\text {max }}\right) \min \left\{\hat{R}_{1, k}^{i, c}, \hat{R}_{2, k}^{i, c^{(1)}}+\hat{R}_{2, k}^{i, c}\right\} . \quad(A-2)
\end{aligned}
$$

Similarly, the EST of the private stream related to the legitimate users, denoted by $E S T_{n, k}^{i, p}$, is expressed by:

$$
E S T_{n, k}^{i, p} \triangleq \hat{R}_{n, k}^{i, p}\left(1-\mathbb{P}\left\{\hat{R}_{n, k}^{i, p} \geq C_{n, k}^{i, p}\right\}\right), \quad(A-3)
$$

$\forall n \in\{1,2\}$, and $\hat{R}_{n, k}^{i, p} \triangleq \theta \log _{2}\left(1+\hat{\gamma}_{n, k}^{i, p}\right)$ therein represents the AIR counterpart. For each user, $E S T_{n, k}^{i, p}$ is limited by the worst-case OP overall the channel realizations, to be able to always detect the corresponding private stream. So, the associated transmission rate of the private stream at the legitimate users is given by (27).

\section{APPENDIX B \\ ProOF OF LEMMA 5}

With the aim of the linearization of (34- $\left.C_{3}\right)$, after substituting the definition of $\hat{R}_{n, k}^{i, p}$ and using some variable transformation, the three extra auxiliary constraints are formulated for $\forall n \in\{1,2\}$, as follows:

$$
\begin{array}{ll}
\theta \beta_{p, n} \geq \alpha_{p, n} & (B-1) \\
\hat{\gamma}_{n, k}^{i, p}(1) \geq \rho_{p, n} & (B-2) \\
1+\rho_{p, n}-2^{\beta_{p, n}} \geq 0 . & (B-3)
\end{array}
$$

To maintain the generality of the problem, the auxiliary constraints have to be defined for ensuring that they would be active at the optimum. Although $(B-3)$ is convex, substituting the definition of $\hat{\gamma}_{n, k}^{i, p}$ into $(B-2)$, it can be represented as the difference of two convex functions (DC decomposition) and thus it is non-convex. Furthermore, $(B-1)$ is also nonconvex due to the nonlinear term $\theta \beta_{p, n}$. To deal with this nonconvexity, we first write the equivalent DC decomposition of $(B-1)$ as:

$$
\begin{gathered}
\alpha_{p, n}+\left(\mathcal{A}\left(\theta, \beta_{p, n}\right)-\mathcal{B}\left(\theta, \beta_{p, n}\right)\right) \quad(B-4) \\
\leq 0, \forall n \in\{1,2\},
\end{gathered}
$$

where $\mathcal{A}\left(\theta, \beta_{p, n}\right) \triangleq \frac{1}{4}\left(\theta-\beta_{p, n}\right)^{2}$ and $\mathcal{B}\left(\theta, \beta_{p, n}\right) \triangleq \frac{1}{4}(\theta+$ $\left.\beta_{p, n}\right)^{2}$. As it can be observed, the non-convexity of $(B-4)$ is caused by $\mathcal{B}\left(\theta, \beta_{p, n}\right)$. Therefore, we replace it by its affine approximation obtained by the first order Taylor expansion at the $m$-th iteration, i.e. $\hat{\mathcal{B}}^{[m]}\left(\theta, \beta_{p, n}\right)$. Subsequently, inserting $\hat{\mathcal{B}}^{[m]}\left(\theta, \beta_{p, n}\right)$ into $(B-4)$, the affine approximation of $(B-1)$ is given by:

$$
\Theta^{[m]}\left(\theta, \beta_{p, n}\right) \geq \alpha_{p, n},
$$

where $\Theta^{[m]}(x, y)$ was introduced in Lemma 5 .

\section{APPENDiX C \\ PROOF OF LEMMA 7}

Inserting the definition of $C_{e, n, k}^{i, c}$ into constraint (34- $C_{5}$ ), with the aim of linearization, we follow the same approaches in Appendix B, resulting in the following additional constraints $\forall n \in\{1,2\},, n \neq j$ : 


$$
\begin{gathered}
\theta \beta_{n, e} \leq \alpha_{n, e}, \\
\hat{\gamma}_{e, n, k}^{i, p} \leq \rho_{n, e}, \\
1+\rho_{n, e}-2^{\beta_{n, e}} \leq 0 .
\end{gathered}
$$

While $(C-3)$ is a non-convex constraint by itself, the nonconvexity of $(B-(1,2))$ is shown by recasting them through DC decomposition. Given this perspective and recalling the functions introduced in Appendix B, the following DC decompositions are rendered for $(C-(1,2))$, respectively:

$$
\begin{array}{cc}
\mathcal{B}\left(\theta, \beta_{n, e}\right)-\mathcal{A}\left(\theta, \beta_{n, e}\right) \leq \alpha_{n, e} & (C-4) \\
\mathcal{M}_{1}(\mathbf{z})-\mathcal{N}_{1}(\mathbf{z}) \leq 0, & (C-5)
\end{array}
$$

where $\mathbf{z}=\left[\mathbf{p}_{n, k}^{i, p}, \mathbf{p}_{k}^{i, c}, \mathbf{p}_{j, k}^{i, p}, \rho_{n, e}\right], \mathcal{M}_{1}(\mathbf{z}) \triangleq \frac{\left|\hat{\mathbf{h}}_{e, k}^{(1) H} \mathbf{p}_{n, k}^{i, p}\right|^{2}}{\rho_{n, e}}$, $\mathcal{N}_{1}(\mathbf{z}) \triangleq\left|\hat{\mathbf{h}}_{e, k}^{(1) H} \mathbf{p}_{k}^{i, c}\right|^{2}+\left|\hat{\mathbf{h}}_{e, k}^{(1) H} \mathbf{p}_{j, k}^{i, p}\right|^{2}+p_{M, k}\left\|\mathbf{w}_{e, k}^{(1)}{ }^{H} \mathbf{C}_{n, k}^{i}\right\|^{2}$ $+\delta^{2}$. While the non-convexity of $(C-1)$ and $(C-2)$, respectively, is caused by $\left.\mathcal{A}\left(\theta, \beta_{n, e}\right)\right)$ and $\mathcal{N}_{1}(\mathbf{z})$, respectively, as shown in $(C-4)$ and $(C-5)$, for $(C-3)$ this is due to the term $2^{\beta_{n, e}}$. So, the non-convex factors have to be replaced at the $m$-th iteration by their first-order Taylor expansion. The Taylor expansion of $\mathcal{A}\left(\theta, \beta_{n, e}\right), \mathcal{N}_{1}(\mathbf{z})$, and $2^{\beta_{n, e}}$ are respectively represented by the operators $\bar{\Theta}^{[m]}(x, y)$, $\Omega^{[m]}(\mathbf{u}, \mathbf{v} ; \mathbf{h})$, and $\Gamma^{[m]}(x)$.

\section{APPENDIX D}

\section{PROOF OF LEMMA 9}

The constraint $\left(31-C_{2}\right)$ can be equivalently rewritten as follows:

$$
\begin{gathered}
C_{e, k}^{i, c} \leq \alpha_{c, e} \leq r_{k}^{i, c}, \\
\left\{\forall \boldsymbol{\Delta} \mathbf{n}_{n, k}^{i} \in \Theta_{\mathbf{h}_{n}}, \forall \Delta \mathbf{f}_{e, k}^{i} \in \Theta_{f_{e}}\right\} .
\end{gathered}
$$$$
(D-1)
$$

Problem $(D-1)$ may be solved by a search over the possible values of CSI uncertainties to find the worst-case. On the other hand, based on what we inferred earlier from (34- $\left.C_{1}\right)$ and (34$C_{6}$ ), the goal is to minimize $C_{e, k}^{i, c}$ by minimizing its ceiling rate $\alpha_{c, e}$. Incorporating this perspective into $(D-1)$, it is expected that this ceiling does not exceed the predefined target rate $r_{k}^{i, c}$, i.e. $C_{e, k}^{i, c} \leq \alpha_{c, e} \leq r_{k}^{i, c}$ for various imperfections. Given this fact, while following the same approach as Appendix B, $(D-1)$ is transformed into some extra artificial constraints, aimed at linearization. Thus, by inserting the definition of $C_{e, k}^{i, c}$ as well as utilizing the same auxiliary variables as introduced in (38) for $\hat{\gamma}_{e, k}^{i, c}{ }^{(1)}, \hat{\gamma}_{e, k}^{i, c}(2)$ along with new auxiliary variables, the problem $(D-1)$ after some trivial manipulation can be recast as:

$$
\begin{array}{ll}
\theta \log _{2}\left(1+\rho_{c, e}^{(1)}\right)+(1-\theta) \log _{2}\left(1+\rho_{c, e}^{(2)}\right) \leq \alpha_{c, e}, & (D-2) \\
\frac{x_{c, e}^{2}}{\sum_{\substack{u_{n, c, e}+p_{M, k} \\
n=1}}^{2} \mathbf{c}_{e, k}^{i} \|^{2}+\delta^{2}} \leq \rho_{c, e}^{(1)}, & (D-3) \\
\frac{\delta_{R}^{2}}{v_{c, e}+p_{M, k}\left\|\mathbf{c}_{e, k}^{i}\right\|^{2}+\delta^{2}} \leq \rho_{c, e}^{(2)}, & (D-4)
\end{array}
$$

$$
\begin{aligned}
& \underset{\Delta \mathbf{n}_{\mathbf{h}_{e, k}^{i}}}{\max }\left|\mathbf{h}_{e, k}^{(1)^{H}} \mathbf{p}_{k}^{i, c}\right| \leq x_{c, e}, \\
& \underset{\Delta \mathbf{n}_{\mathbf{h}_{e, k}^{i}}}{\min }\left|\mathbf{h}_{e, k}^{(1)^{H}} \mathbf{p}_{n, k}^{i, p}\right|^{2} \geq u_{n, c, e}, n \in\{1,2\} \\
& \underset{\boldsymbol{\Delta n}_{\mathbf{h}^{i}, k}}{\min } p_{j}\left|\mathbf{h}_{e, k}^{i}{ }^{H} \mathbf{p}_{z}\right|^{2} \geq v_{c, e}
\end{aligned}
$$

$\forall \boldsymbol{\Delta} \mathbf{n}_{n, k}^{i} \in \Theta_{\mathbf{h}_{n}}$ and $\triangle \mathbf{f}_{e, k}^{i} \in \Theta_{f_{e}}$. However, $(D-(2: 7))$ are still non-convex. The non-convex constraint $(D-2)$ is the same we have already addressed in (38-(I)) and we neglect rewriting it here. To circumvent the non-convexity of $(D-$ 3), two extra artificial constraints (39-(III)) and (39-(IV)) are introduced. Similarly, as for $(D-4)$, we have equivalently derived (39-(III)) and (39-(IV)).

To handle the non-convex constraints $(D-(5: 7))$, we exploit the following proposition.

Proposition 13. For the terms $\mathcal{F}_{1}(\Delta \mathbf{h}) \triangleq\left|(\hat{\mathbf{h}}+\Delta \mathbf{h})^{H} \mathbf{u}\right|$ and $\mathcal{F}_{2}(\boldsymbol{\Delta} \mathbf{h}) \triangleq\left|(\hat{\mathbf{h}}+\boldsymbol{\Delta} \mathbf{h})^{H} \mathbf{u}\right|^{2}$ with norm-bounded variable $\|\mathbf{\Delta} \mathbf{h}\|_{2} \leq \sigma$, the following minimizer and maximizer can be presented:

$$
\begin{array}{rlr}
\min _{\|\mathbf{\Delta}\|_{2} \leq \sigma} \mathcal{F}_{1}(\boldsymbol{\Delta} \mathbf{h}) & =\left|\hat{\mathbf{h}}^{H} \mathbf{u}\right|+\sigma\|\mathbf{u}\|_{2}, & (D-9) \\
\min _{\|\mathbf{\Delta}\|_{2} \leq \sigma} \mathcal{F}_{2}(\boldsymbol{\Delta} \mathbf{h}) & =\operatorname{Tr}[(\hat{\mathbf{H}}-\mu \mathbf{I}) \mathbf{U}], & (D-10) \\
\max _{\|\mathbf{\Delta}\|_{2} \leq \sigma} \mathcal{F}_{2}(\boldsymbol{\Delta} \mathbf{h}) & =\operatorname{Tr}[(\hat{\mathbf{H}}+\mu \mathbf{I}) \mathbf{U}], & (D-11) \\
\text { where } \hat{\mathbf{H}} \triangleq \hat{\mathbf{h}}^{H}, \mathbf{U} \triangleq \mathbf{u u}^{H}, \mu \triangleq \sigma^{2}+2 \sigma\|\hat{\mathbf{h}}\|_{2} . &
\end{array}
$$

Proof: Please refer to [43].

Using proposition 1 , we can relax the right-side of $(D-$ $(5: 7))$, leading to respectively as presented in (39-(VI))-(39$(\mathrm{IX}))$.

\section{APPENDIX E \\ PROOF OF LEMMA 10}

Based on what was discussed in Lemma 9, after some trivial manipulation, the following artificial constraints are obtained $\forall n, j \in\{1,2\}, n \neq j$ :

$$
\begin{gathered}
\frac{\left|\hat{\mathbf{h}}_{n, k}^{i^{H}} \mathbf{p}_{n, k}^{i, p}\right|^{2}}{\gamma_{p, n}}-\underbrace{\left|\hat{\mathbf{h}}_{n, k}^{i^{H}} \mathbf{p}_{j, k}^{i, p}\right|^{2}}_{\mathcal{K}_{1}}-p_{M, k}\left\|\mathbf{c}_{n, k}^{i}\right\|^{2}-\delta^{2} \leq 0 \quad(E-1) \\
\gamma_{p, n}-\underbrace{\frac{t_{p, n}}{\omega_{p, j}} \leq 0,}_{\mathcal{K}_{2}}(E-2) \\
t_{p, n}+\kappa_{n} \operatorname{Tr}\left[\mathbf{p}_{n, k}^{i, p}\right]-\underbrace{\operatorname{Tr}\left[\hat{\mathbf{H}}_{n, k} \mathbf{P}_{n, k}^{i, p}\right]}_{\mathcal{K}_{3}} \leq 0 \quad(E-3) \\
v_{j, p, n}+p_{M, k}\left\|\mathbf{c}_{n, k}^{i}\right\|^{2}-\omega_{p, j} \leq 0 \\
\operatorname{Tr}\left[\left(\hat{\mathbf{H}}_{n, k}+\kappa_{n} \mathbf{I}\right) \mathbf{p}_{j, k}^{i, p}\right]-v_{j, p, n} \leq 0 .
\end{gathered}
$$


After DC representation, it can be explicitly observed that $(E-(1: 3))$ are still non-convex, since they are represented by the difference of the convex terms $\left.\mathcal{K}_{i}\right|_{i=1} ^{3}$ that have to be replaced at the $m$-th iteration by their first order Taylor expansion, respectively, given by the operators $\Psi^{[m]}\left(\mathbf{p}_{j, k}^{i, p}, 1 ; \hat{\mathbf{h}}_{n, k}^{i}\right)$, $\mathcal{T}^{[m]}\left(t_{p, n}, \omega_{p, j}\right), \Psi^{[m]}\left(\mathbf{p}_{n, k}^{i, p}, 1 ; \hat{\mathbf{h}}_{n, k}^{i}\right)$.

\section{REFERENCES}

[1] Y. Zou, J. Zhu, X. Wang and L. Hanzo, "A Survey on Wireless Security: Technical Challenges, Recent Advances, and Future Trends," in Proceedings of the IEEE, vol. 104, no. 9, pp. 1727-1765, Sept. 2016.

[2] Chen, Xiaoming, Derrick Wing Kwan Ng, Wolfgang H. Gerstacker, and Hsiao-Hwa Chen. "A survey on multiple-antenna techniques for physical layer security." IEEE Communications Surveys \& Tutorials 19, no. 2 (2016): 1027-1053.

[3] Z. Kong, J. Song, C. Wang, H. Chen and L. Hanzo, "Hybrid Analog Digital Precoder Design for Securing Cognitive Millimeter Wave Networks," in IEEE Transactions on Information Forensics and Security, 2020.

[4] Z. Kong, S. Yang, D. Wang and L. Hanzo, "Robust Beamforming and Jamming for Enhancing the Physical Layer Security of Full Duplex Radios," in IEEE Transactions on Information Forensics and Security, vol. 14, no. 12, pp. 3151-3159, Dec. 2019.

[5] Y. Yang, M. Ma, S. Aïssa and L. Hanzo, "Physical-Layer Secret Key Generation via CQI-Mapped Spatial Modulation in Multi-Hop Wiretap Ad-Hoc Networks," in IEEE Transactions on Information Forensics and Security, vol. 16, pp. 1322-1334, 2021.

[6] A. Mukherjee, S. A. A. Fakoorian, J. Huang, and A. L. Swindlehurst, "Principles of physical layer security in multiuser wireless networks: A survey," IEEE Communications Surveys \& Tutorials, vol. 16, no. 3, pp. 1550-1573, 2014.

[7] M. Moradikia, H. Bastami, A. Kuhestani, H. Behroozi, and L. Hanzo, "Cooperative secure transmission relying on optimal power allocation in the presence of untrusted relays, a passive eavesdropper and hardware impairments," IEEE Access, vol. 7, pp. 116 942-116 964, 2019.

[8] M. Moradikia, S. Mashdour and A. Jamshidi, "Joint optimal power allocation cooperative beamforming and jammer selection design to secure untrusted relaying network", Trans. Emerg. Telecommun. Technol, vol. 29, no. 3, pp. e3276, 2018.

[9] Mashdour, Saeed, Majid Moradikia, and Phee Lep Yeoh. "Secure mmWave communications with imperfect hardware and uncertain eavesdropper location." Transactions on Emerging Telecommunications Technologies 31, no. 10 (2020): e4016.

[10] J. Tang, L. Jiao, K. Zeng, H. Wen and K. -Y. Qin, "Physical Layer Secure MIMO Communications Against Eavesdroppers With Arbitrary Number of Antennas," in IEEE Transactions on Information Forensics and Security, vol. 16, pp. 466-481, 2021, doi: 10.1109/TIFS.2020.3015548.

[11] M. Dai, B. Clerckx, D. Gesbert, and G. Caire, "A rate splitting strategy for massive MIMO with imperfect CSIT," IEEE Transactions on Wireless Communications, vol. 15, no. 7, pp. 4611-4624, 2016.

[12] H. Zhang, J. Zhang and K. Long, "Energy Efficiency Optimization for NOMA UAV Network With Imperfect CSI," in IEEE Journal on Selected Areas in Communications, vol. 38, no. 12, pp. 2798-2809, Dec. 2020, doi: 10.1109/JSAC.2020.3005489.

[13] Y. Liu, Y. Yang, L. -L. Yang and L. Hanzo, "Physical Layer Security of Spatially Modulated Sparse-Code Multiple Access in Aeronautical Ad-hoc Networking," in IEEE Transactions on Vehicular Technology, vol. 70, no. 3, pp. 2436-2447, March 2021.

[14] Shu, Feng, Xiaomin Wu, Jun Li, Riqing Chen, and Branka Vucetic. "Robust synthesis scheme for secure multi-beam directional modulation in broadcasting systems." IEEE Access 4 (2016): 6614-6623.

[15] Lin, S-C., T-H. Chang, Y-W. Hong, and C-Y. Chi. "On the impact of quantized channel direction feedback in multiple-antenna wiretap channels." In 2010 IEEE International Conference on Communications, pp. 1-5. IEEE, 2010.

[16] B. Li, Z. Fei, and Y. Zhang, "UAV communications for 5G and beyond: Recent advances and future trends," IEEE Internet of Things Journal, vol. 6, no. 2, pp. 2241-2263, 2018.

[17] Y. Wu, Y. He, L. P. Qian, J. Huang, and X. Shen, "Optimal resource allocations for mobile data offloading via dual-connectivity," IEEE Transactions on Mobile Computing, vol. 17, no. 10, pp. 2349-2365, 2018.
[18] Bai, Tong, Jingjing Wang, Yong Ren, and Lajos Hanzo. "Energy-efficient computation offloading for secure UAV-edge-computing systems." IEEE Transactions on Vehicular Technology 68, no. 6 (2019): 6074-6087.

[19] Nasser, Ahmed, Osamu Muta, and Maha Elsabrouty. "Cross-tier interference management scheme for downlink mMIMIO-NOMA HetNet." In 2019 IEEE 89th Vehicular Technology Conference (VTC2019-Spring), pp. 1-5. IEEE, 2019.

[20] M. Alzenad, A. El-Keyi, and H. Yanikomeroglu, "3-D placement of an unmanned aerial vehicle base station for maximum coverage of users with different QoS requirements," IEEE Wireless Communications Letters, vol. 7, no. 1, pp. 38-41, 2017.

[21] M. Srinivasan, S. Gopi, S. Kalyani, X. Huang and L. Hanzo, "AirplaneAided Integrated Next-Generation Networking," in IEEE Transactions on Vehicular Technology.

[22] L. Xiao, Y. Xu, D. Yang and Y. Zeng, "Secrecy Energy Efficiency Maximization for UAV-Enabled Mobile Relaying," in IEEE Transactions on Green Communications and Networking, vol. 4, no. 1, pp. 180-193, March 2020.

[23] Xu, Kaidi, Ming-Min Zhao, Yunlong Cai, and Lajos Hanzo. "Lowcomplexity joint power allocation and trajectory design for UAV-enabled secure communications with power splitting." IEEE Transactions on Communications 69, no. 3 (2020): 1896-1911.

[24] Tang, Na, Hongying Tang, Baoqing Li, and Xiaobing Yuan. "Cognitive NOMA for UAV-enabled secure communications: Joint 3D trajectory design and power allocation." IEEE Access 8 (2020): 159965-159978.

[25] Mamaghani, Milad Tatar, and Yi Hong. "Intelligent Trajectory Design for Secure Full-Duplex MIMO-UAV Relaying Against Active Eavesdroppers: A Model-Free Reinforcement Learning Approach." IEEE Access 9 (2020): 4447-4465.

[26] Y. Chen and Z. Zhang, "UAV-aided secure transmission in MISOME wiretap channels with imperfect CSI,' IEEE Access, vol. 7, pp. 98107 98 121, 2019.

[27] Y. Mao, B. Clerckx, and V. O. Li, "Rate-splitting multiple access for downlink communication systems: bridging, generalizing, and outperforming SDMA and NOMA," EURASIP journal on wireless communications and networking, vol. 2018, no. 1, p. 133, 2018

[28] J. Zhang, B. Clerckx, J. Ge and Y. Mao, "Cooperative Rate Splitting for MISO Broadcast Channel With User Relaying, and Performance Benefits Over Cooperative NOMA," in IEEE Signal Processing Letters, vol. 26, no. 11, pp. 1678-1682, Nov. 2019.

[29] H. Fu, S. Feng, W. Tang and D. W. K. Ng, "Robust Secure Beamforming Design for Two-User Downlink MISO Rate-Splitting Systems," in IEEE Transactions on Wireless Communications, vol. 19, no. 12, pp. 83518365, Dec. 2020

[30] P. Li, M. Chen, Y. Mao, Z. Yang, B. Clerckx, and M. Shikh-Bahaei, "Cooperative Rate-Splitting for secrecy sum-rate enhancement in multi antenna broadcast channels," arXiv preprint arXiv:2006.02555, 2020.

[31] Sun, Yan, Derrick Wing Kwan Ng, Jun Zhu, and Robert Schober. "Robust and secure resource allocation for full-duplex MISO multicarrier NOMA systems." IEEE Transactions on Communications 66, no. 9 (2018): 4119-4137.

[32] Y. Li, M. Jiang, Q. Zhang, Q. Li, and J. Qin, "Secure beamforming in downlink MISO non-orthogonal multiple access systems," IEEE Trans. Veh. Technol., vol. 66, no. 8, pp. 7563-7567, Aug. 2017.

[33] C. Hao and B. Clerckx, "MISO networks with imperfect CSIT: A topological rate-splitting approach," IEEE Transactions on Communications, vol. 65, no. 5, pp. 2164-2179, 2017.

[34] H. Bastami, M. Moradikia, H. Behroozi, R. C. de Lamare, A. Abdelhadi, and Z. Ding, "Secrecy rate maximization for hardware impaired untrusted relaying network with deep learning," http://arxiv.org/abs/2101.02749, 2021

[35] Yen, Kai, and Lajos Hanzo. "Antenna-diversity-assisted geneticalgorithm-based multiuser detection schemes for synchronous CDMA systems." IEEE Transactions on Communications 51, no. 3 (2003): 366370.

[36] Xu, Chong, Rob G. Maunder, Lie-Liang Yang, and Lajos Hanzo. "Nearoptimum multiuser detectors using soft-output ant-colony-optimization for the DS-CDMA uplink." IEEE Signal Processing Letters 16, no. 2 (2009): 137-140.

[37] Yetgin, Halil, Kent Tsz Kan Cheung, and Lajos Hanzo. "Multi-objective routing optimization using evolutionary algorithms." In 2012 IEEE Wireless Communications and Networking Conference (WCNC), pp. 3030-3034. IEEE, 2012.

[38] Jiang, Ming, Jos Akhtman, and Lajos Hanzo. "Iterative joint channel estimation and multi-user detection for multiple-antenna aided OFDM systems." IEEE Transactions on Wireless Communications 6, no. 8 (2007): 2904-2914. 
[39] J. Cui, H. Yetgin, D. Liu, J. Zhang, S. X. Ng and L. Hanzo, "TwinComponent Near-Pareto Routing Optimization for AANETs in the North-Atlantic Region Relying on Real Flight Statistics," in IEEE Open Journal of Vehicular Technology.

[40] Omri and M. O. Hasna, "Physical Layer Security Analysis of UAV Based Communication Networks," 2018 IEEE 88th Vehicular Technology Conference (VTC-Fall), Chicago, IL, USA, 2018, pp. 1-6.

[41] E. Boshkovska, D. W. K. Ng, N. Zlatanov, A. Koelpin and R. Schober, "Robust resource allocation for MIMO wireless powered communication networks based on a non-linear EH model," in IEEE Transactions on Communications, vol. 65, no. 5, pp. 1984-1999, May 2017.

[42] M. Grant and S. Boyd. (2016). "CVX: MATLAB Software for Disciplined Convex Programming," [Online]. Available: http://cvxr.com/cvx.

[43] E. A. Gharavol, Y. Liang and K. Mouthaan, "Robust Downlink Beamforming in Multiuser MISO Cognitive Radio Networks With Imperfect Channel-State Information," in IEEE Transactions on Vehicular Technology, vol. 59, no. 6, pp. 2852-2860, July 2010.

[44] B. Clerckx et al., "Is NOMA Efficient in Multi-Antenna Networks? A Critical Look at Next Generation Multiple Access Techniques," in IEEE Open Journal of the Communications Society, vol. 2, pp. 1310-1343, 2021, doi: 10.1109/OJCOMS.2021.3084799.

[45] J. Tang, M. Dabaghchian, K. Zeng and H. Wen, "Impact of Mobility on Physical Layer Security Over Wireless Fading Channels," in IEEE Transactions on Wireless Communications, vol. 17, no. 12, pp. 78497864, Dec. 2018, doi: 10.1109/TWC.2018.2868935.

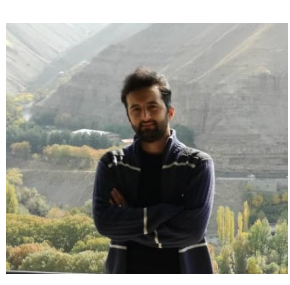

Hamed Bastami received the M.Sc. degree in Electrical Engineering from the University of Tehran, Tehran, Iran, in 2014. He is currently a Ph.D. student with the Department of Electrical Engineering, Sharif University of Technology. His research interests lie in the areas of physical-layer security (PLS) of wireless communications with special emphasis on machine learning, deep-based resource management, PLS in wireless communication and fog radio access networks.

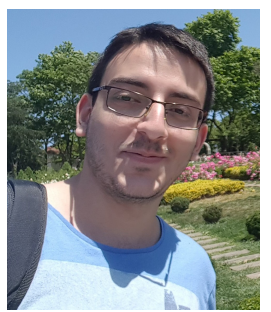

Mehdi Letafati received his B.Sc degree in Electrical Engineering from Sharif University of Technology, Tehran, Iran, in 2019. Currently, he is an M.S. student of Communications at Sharif University of Technology. Since 2015, he has been a student member of Iran's National Elites Foundation. His research interests include learning-assisted physicallayer security for wireless networks, cooperative communications for the Internet of Things, privacy in data science, and optimization methods. He was honored to be ranked 4th among all participants in the Iranian Nationwide University Entrance Exam in 2015. He is currently a reviewer of the IEEE Internet of Things Journal and IEEE Wireless Communication Letters.

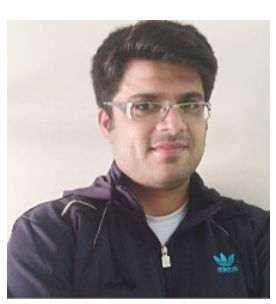

Majid Moradikia was born in 1986. He received the Ph.D. degree in Telecommunication system engineering at the Department of Electrical and Electronics Engineering, Shiraz University of Technology, Shiraz, Iran. He currently works as a postdoctoral research fellow at the Engineering Technology Department at University of Houston, Texas, USA. His main research interests lie within the area of physical-layer security of wireless communications, Internet of Things, millimeter-wave communication systems, massive MIMO systems.

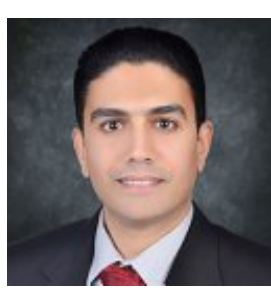

Ahmed Abdelhadi is an Assistant Professor at the University of Houston. Before joining UH, he was a Research Assistant Professor at Virginia Tech. He received his Ph.D. in Electrical and Computer Engineering from the University of Texas at Austin in 2011. He was a member in Wireless Networking and Communications Group (WNCG) and Laboratory of Informatics, Networks and Communications (LINC) group during his Ph.D. In 2012, he joined Bradley Department of Electrical and Computer Engineering and Hume Center for National Security and Technology at Virginia Tech. He was a faculty member of Wireless @ Virginia Tech. His research interests are in the areas of wireless communications and networks, artificial intelligence, cyber physical systems, and security. Dr. Abdelhadi coauthored more than 80 journal and conference papers, and 7 books in these research topics. He is a senior member of IEEE. His book Cellular Communications Systems in Congested Environments is bookplated as Virginia Tech Provost's Honor Book and his book Resource Allocation with Carrier Aggregation in Cellular Networks is featured in the 13th Annual Virginia Tech Authors Recognition Event. He received Silver Contribution Award from IEEE International Conference on Computing, Networking and Communications (ICNC), Best Paper Award from IEEE International Symposium on Systems Engineering (ISSE), and Outstanding Paper Award from IEEE International Conference of Advanced Communications Technology (ICACT).

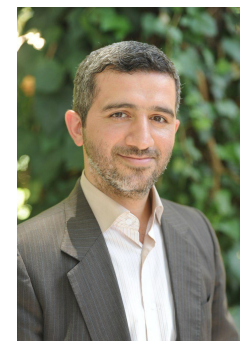

Hamid Behroozi (S'04-M'08) received the B.Sc. degree in Electrical Engineering from the University of Tehran, Tehran, Iran, in 2000, the M.Sc. degree in Electrical Engineering from Sharif University of Technology, Tehran, in 2003, and the Ph.D. degree in Electrical Engineering from Concordia University, Montreal, QC, Canada, in 2007. From 2007 to 2010, he was a Postdoctoral Fellow with the Department of Mathematics and Statistics, Queens University, Kingston, ON, Canada. He is currently an Associate Professor with the Department of Electrical Engineering, Sharif University of Technology, Tehran. His research interests include information theory, joint source-channel coding, artificial intelligence in signal processing and data science, and cooperative communications. Dr. Behroozi was the recipient of several academic awards, including Ontario Postdoctoral Fellowship awarded by the Ontario Ministry of Research and Innovation (MRI), Quebec Doctoral Research Scholarship awarded by the Government of Quebec (FQRNT), Hydro Quebec Graduate Award, and Concordia University Graduate Fellowship.

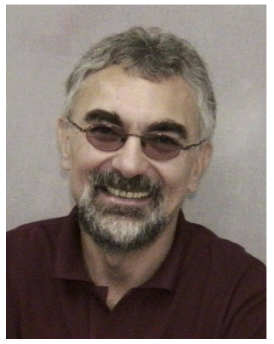

Lajos Hanzo (Fellow, IEEE) received the master's and $\mathrm{Ph} . \mathrm{D}$. degrees from the Technical University (TU) of Budapest in 1976 and 1983, respectively, the D.Sc. degree from the University of Southampton in 2004, and the Honorary Doctorates from the TU of Budapest in 2009 and from the University of Edinburgh in 2015. He is currently a Foreign Member of the Hungarian Academy of Sciences and the former Editor-in-Chief of IEEE Press. He has served several terms as Governor of both IEEE ComSoc and of VTS. He has published more than 1900 contributions at IEEE Xplore, 19 Wiley-IEEE Press books and has helped the fast-track career of 123 Ph.D. students. More than 40 of them are Professors at various stages of their careers in academia and many of them are leading scientists in the wireless industry. He is a fellow of the Royal Academy of Engineering (REng.), IET, and EURASIP. 